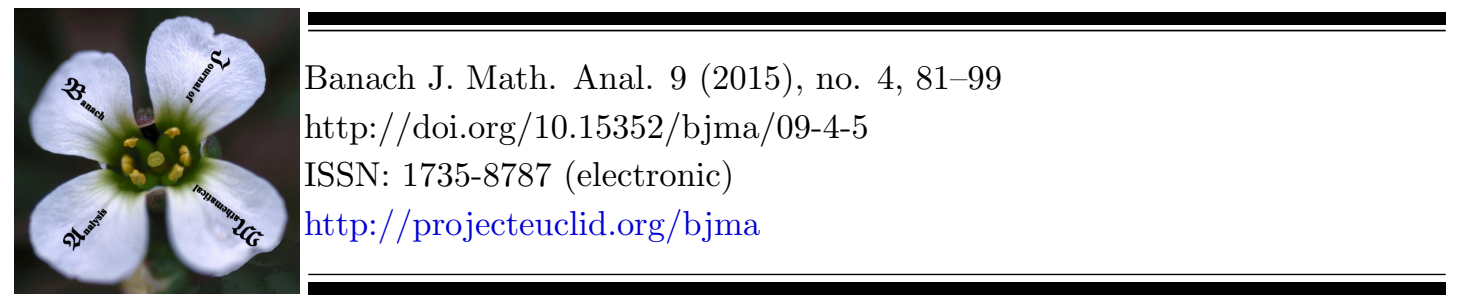

\title{
ON REDUNDANCY, DILATIONS AND CANONICAL DUALS OF $g$-FRAMES IN HILBERT SPACES
}

\author{
XUNXIANG GUO*
}

Communicated by M. S. Moslehian

\begin{abstract}
In Hilbert spaces, the redundancy property of $g$-frames is different from that of frames, and the dilation theory is interesting and important in many mathematical fields. In this paper, we study the redundancy and dilations of $g$-frames in Hilbert spaces. First, we characterize $g$-Riesz bases and exact $g$-frames under some constraints, then obtain some dilation results for (normalized tight) $g$-frames, and give some properties about them. Finally we prove some interesting properties on the canonical duals of $g$-frames.
\end{abstract}

\section{INTRODUCTION}

Throughout this paper, we use $\mathcal{N}$ to denote the set of natural numbers and $\mathcal{C}$ to denote the set of complex numbers.

A sequence $\left\{f_{i}\right\}_{i \in \mathcal{N}}$ of elements in a Hilbert space $H$ is called a frame for $H$ if there are constants $A, B>0$ so that

$$
A\|f\|^{2} \leq \sum_{i \in \mathcal{N}}\left|\left\langle f, f_{i}\right\rangle\right|^{2} \leq B\|f\|^{2}, \text { for all } f \in H .
$$

The numbers $A$ and $B$ are called the lower and upper frame bound respectively. The frame is a tight frame if $A=B$ and a normalized tight frame if $A=B=1$.

The concept of frame first appeared in the late 40's and early 50's (see [6][21][24]). The development and study of wavelet theory during the last decades also brought

Date: Received: Jun. 4, 2014; Revised: Dec. 11, 2014; Accepted: Dec. 24, 2014.

* Corresponding author.

2010 Mathematics Subject Classification. Primary 46C05; Secondary 42C15.

Key words and phrases. $g$-frame, $g$-Riesz basis, exact $g$-frame, redundancy, dilation. 
new ideas and attentions to frames because of their close connections. There are many related references on this topic, see [4][7][14][15][19].

In [22], Sun raised the concept of $g$-frame as follows, which generalizes the concept of frame extensively. A sequence $\left\{\Lambda_{i} \in B\left(H, H_{i}\right): i \in \mathcal{N}\right\}$ is called a $g$ frame for $H$ with respect to $\left\{H_{i}: i \in \mathcal{N}\right\}$, which is a sequence of closed subspaces of a Hilbert space $K$, if there exist two positive constants $A$ and $B$ such that for any $f \in H$

$$
A\|f\|^{2} \leq \sum_{i \in \mathcal{N}}\left\|\Lambda_{i} f\right\|^{2} \leq B\|f\|^{2},
$$

where $A$ is called the lower frame bound and $B$ is called the upper frame bound. The largest lower frame bound and the smallest upper frame bound are called the optimal lower frame bound and the optimal upper frame bound respectively. We simply call $\left\{\Lambda_{i}: i \in \mathcal{N}\right\}$ a $g$-frame for $H$ whenever the space sequence $\left\{H_{i}: i \in\right.$ $\mathcal{N}\}$ is clear. The tight $g$-frame, normalized tight $g$-frame are defined similarly. We call $\left\{\Lambda_{i}: i \in \mathcal{N}\right\}$ a $g$-frame sequence, if it is a $g$-frame for $\overline{\operatorname{span}}\left\{\Lambda_{i}^{*}\left(H_{i}\right)\right\}_{i \in \mathcal{N}}$. We call $\left\{\Lambda_{i}: i \in \mathcal{N}\right\}$ a $g$-Bessel sequence, if only the right inequality is satisfied. A $g$-frame $\left\{\Gamma_{j}: j \in \mathcal{N}\right\}$ for $H$ is called an alternate dual $g$-frame of $\left\{\Lambda_{j}: j \in \mathcal{N}\right\}$, if for every $f \in H$ we have

$$
f=\sum_{j \in \mathcal{N}} \Lambda_{j}^{*} \Gamma_{j} f=\sum_{j \in \mathcal{N}} \Gamma_{j}^{*} \Lambda_{j} f .
$$

If $\left\{\Lambda_{j}: j \in \mathcal{N}\right\}$ is a $g$-frame for $H$, then the operator $S \in B(H)$ such that

$$
S f=\sum_{j \in \mathcal{N}} \Lambda_{j}^{*} \Lambda_{j} f, \forall f \in H
$$

is called the fame operator associated with $\left\{\Lambda_{j}: j \in \mathcal{N}\right\}$. It is well-known that $\left\{\Lambda_{j} S^{-1}: j \in \mathcal{N}\right\}$ is an alternate dual $g$-frame of $\left\{\Lambda_{j}: j \in \mathcal{N}\right\}$, which is also called the canonical dual $g$-frame associated with $\left\{\Lambda_{j}: j \in \mathcal{N}\right\}$ and we denote it as $\left\{\widetilde{\Lambda}_{j}: j \in \mathcal{N}\right\}$. Recently, $g$-frames in Hilbert spaces have been studied intensively, for more details see [8][16][20][23][25] and the references therein.

$G$-frames generalize frames in Hilbert spaces extensively. It has been shown that $g$-frames and frames share many similar properties. But it was pointed out in [22] that the redundancy property is one property where $g$-frames and frames behave differently. It is well-known that the class of exact frames is same as the class of Riesz bases in Hilbert spaces, but the class of exact $g$-frames is different from the class of $g$-Riesz bases. So there is an interesting problem: under what conditions these two classes coincide? Recently, in [18], the authors studied such problem. And in [26], the authors studied the excess of $g$-frames. In this paper we give some new conditions such that the above classes are the same. On the other hand, dilation theory is one of interesting and active theories in many branches of mathematics. For general dilation theory see [1][12]. In [5][13][14], the authors studied the dilation theory on frames in Hilbert spaces and Banach spaces. In this paper, we also consider the dilations of $g$-frames in Hilbert spaces. Finally, we know that the canonical duals of $g$-frames play an important role in the analysis and reconstruction of vectors in Hilbert spaces, so we prove some 
important properties of the canonical duals of $g$-frame at the end section of this paper.

\section{Preliminary DeFinitions And LEMmas}

In this section, we introduce some basic definitions and lemmas which are necessary for the following sections.

Definition 2.1. A $g$-frame $\left\{\Lambda_{j}: j \in \mathcal{N}\right\}$ with respect to $\left\{H_{j}: j \in \mathcal{N}\right\}$ for $H$ is called an exact $g$-frame if for any fixed $j_{0} \in \mathcal{N}$, the sequence $\left\{\Lambda_{j}: j \in \mathcal{N}, j \neq j_{0}\right\}$ is not a $g$-frame for $H$ with respect to $\left\{H_{j}: j \in \mathcal{N}, j \neq j_{0}\right\}$.

Definition 2.2. Suppose that $\Lambda_{j}, \Gamma_{j} \in B\left(H, H_{j}\right)$ for any $j \in \mathcal{N}$. If for any $i, j \in \mathcal{N}$ and any $g_{i} \in H_{i}, g_{j} \in H_{j},\left\langle\Lambda_{i}^{*} g_{i}, \Gamma_{j}^{*} g_{j}\right\rangle=\delta_{i, j}\left\langle g_{i}, g_{j}\right\rangle$, then we call that $\left\{\Lambda_{j}: j \in \mathcal{N}\right\}$ and $\left\{\Gamma_{j}: j \in \mathcal{N}\right\}$ are g-biorthonormal.

Definition 2.3. Let $\Lambda_{i} \in B\left(H, H_{i}\right), i \in \mathcal{N}$.

(i). If $\left\{f: \Lambda_{i} f=0, i \in \mathcal{N}\right\}=\{0\}$, then we say that $\left\{\Lambda_{i}: i \in \mathcal{N}\right\}$ is $g$-complete.

(ii). If $\left\{\Lambda_{i}: i \in \mathcal{N}\right\}$ is $g$-complete and there are positive constants $A$ and $B$ such that for any finite subset $J \subset \mathcal{N}$ and $g_{j} \in H_{j}, j \in J$,

$$
A \sum_{j \in J}\left\|g_{j}\right\|^{2} \leq\left\|\sum_{j \in J} \Lambda_{j}^{*} g_{j}\right\|^{2} \leq B \sum_{j \in J}\left\|g_{j}\right\|^{2}
$$

then we say that $\left\{\Lambda_{i}: i \in \mathcal{N}\right\}$ is a $g$-Riesz basis for $H$ with respect to $\left\{H_{i}: i \in\right.$ $\mathcal{N}\}$.

(iii). We say that $\left\{\Lambda_{i}: i \in \mathcal{N}\right\}$ is a $g$-orthonormal basis for $H$ with respect to $\left\{H_{i}: i \in \mathcal{N}\right\}$ if it satisfies the following:

$$
\begin{gathered}
\left\langle\Lambda_{i}^{*} g_{i}, \Lambda_{j}^{*} g_{j}\right\rangle=\delta_{i j}\left\langle g_{i}, g_{j}\right\rangle, \forall i, j \in \mathcal{N}, g_{i} \in H_{i}, g_{j} \in H_{j}, \\
\sum_{j \in \mathcal{N}}\left\|\Lambda_{j} f\right\|^{2}=\|f\|^{2}, \forall f \in H .
\end{gathered}
$$

Definition 2.4. $\left\{\Lambda_{j} \in B\left(H, H_{j}\right)\right\}_{j=1}^{\infty}$ is finitely g-linearly independent with respect to $\left\{H_{j}\right\}$ if for any $n \in \mathcal{N}, \sum_{j=1}^{n} \Lambda_{j}^{*} g_{j}=0$ implies $g_{j}=0$, where $g_{j} \in H_{j}(j=$ $1,2, \cdots, n)$.

Definition 2.5. $\left\{\Lambda_{j} \in B\left(H, H_{j}\right)\right\}_{j=1}^{\infty}$ is g-linearly independent with respect to $\left\{H_{j}\right\}$ if $\sum_{j=1}^{\infty} \Lambda_{j}^{*} g_{j}=0$ implies $g_{j}=0$, where $g_{j} \in H_{j}(j=1,2, \cdots)$.

Definition 2.6. $\left\{\Lambda_{j} \in B\left(H, H_{j}\right)\right\}_{j=1}^{\infty}$ is g-minimal with respect to $\left\{H_{j}\right\}$ if for any sequence $\left\{g_{j}: j \in \mathcal{N}\right\}$ with $g_{j} \in H_{j}$ and any $m \in \mathcal{N}$ with $g_{m} \neq 0$, we have $\Lambda_{m}^{*} g_{m} \notin \overline{\operatorname{span}}_{i \neq m}\left\{\Lambda_{i}^{*} g_{i}\right\}$.

Remark 2.7. It is obvious that any $g$-frame is $g$-complete and any $g$-orthonormal basis is a normalized tight $g$-frame.

Definition 2.8. We call $\left\{\Lambda_{j} \in B\left(H, H_{j}\right)\right\}_{j=1}^{\infty}$ a $g$-basis for $H$ with respect to $\left\{H_{j}\right\}$ if for any $x \in H$ there is a unique sequence $\left\{g_{j}\right\}$ with $g_{j} \in H_{j}$ such that $x=\sum_{j=1}^{\infty} \Lambda_{j}^{*} g_{j}$. 
Definition 2.9. Two $g$-frames $\left\{\Lambda_{j}: j \in \mathcal{N}\right\}$ and $\left\{\Gamma_{j}: j \in \mathcal{N}\right\}$ for $H$ are called similar if there exists an invertible operator $T \in B(H)$ such that $\Lambda_{j}=\Gamma_{j} T$ for any $j \in \mathcal{N}$.

The following lemma tells us that $g$-Riesz bases for a Hilbert space $H$ are precisely the sequences of operators which are similar to $g$-orthonormal bases for $H$.

Lemma 2.10. [22] A sequence $\left\{\Lambda_{j}: j \in \mathcal{N}\right\}$ is a g-Riesz basis for $H$ with respect to $\left\{H_{j}: j \in \mathcal{N}\right\}$ if and only if there is a g-orthonormal basis $\left\{\theta_{j}: j \in \mathcal{N}\right\}$ for $H$ and a bounded invertible linear operator $T$ on $H$ such that $\Lambda_{j}=\theta_{j} T, j \in \mathcal{N}$.

The following lemma is from [9], which establishes a useful identity on $g$-frames and some criterions used to verify some redundancy related properties of $g$-frames. Since it is published in a Chinese journal, we include its detailed proof for the sake of convenience.

Lemma 2.11. [9] Suppose $\left\{\Lambda_{j} \in B\left(H, H_{j}\right): j \in \mathcal{N}\right\}$ is a g-frame for $H$ with frame bounds $A$ and $B, g_{j} \in H_{j}, \forall j \in \mathcal{N}$. Then

(1) $\sum_{j \neq m}\left\|\widetilde{\Lambda}_{j} \Lambda_{m}^{*} g_{m}\right\|^{2}=\frac{1}{2}\left[\left\|g_{m}\right\|^{2}-\left\|\widetilde{\Lambda}_{m} \Lambda_{m}^{*} g_{m}\right\|^{2}-\left\|g_{m}-\widetilde{\Lambda}_{m} \Lambda_{m}^{*} g_{m}\right\|^{2}\right]$.

(2) If $\widetilde{\Lambda}_{m} \Lambda_{m}^{*}=I_{H_{m}}$, then $\widetilde{\Lambda}_{j} \Lambda_{m}^{*}=0$ for $j \neq m$. So $\left\{\Lambda_{j}: j \in \mathcal{N}\right\}$ and $\left\{\widetilde{\Lambda}_{j}: j \in\right.$ $\mathcal{N}\}$ are g-biorthonormal if and only if $\widetilde{\Lambda}_{m} \Lambda_{m}^{*}=I_{H_{m}}, \forall m \in \mathcal{N}$.

(3) If $\operatorname{ker}\left(I_{H_{m}}-\widetilde{\Lambda}_{m} \Lambda_{m}^{*}\right) \neq 0$ or $\overline{\operatorname{range}}\left(I_{H_{m}}-\widetilde{\Lambda}_{m} \Lambda_{m}^{*}\right) \neq H_{m}$, then $\left\{\Lambda_{j}\right\}_{j \neq m}$ is not g-complete.

(4) If $I_{H_{m}}-\Lambda_{m} \widetilde{\Lambda}_{m}^{*}$ is surjective or $I_{H_{m}}-\widetilde{\Lambda}_{m} \Lambda_{m}^{*}$ is surjective, then $\left\{\Lambda_{j}\right\}_{j \neq m}$ is a g-frame.

Proof. (1) Since $\left\{\Lambda_{j}: j \in \mathcal{N}\right\}$ is a $g$-frame for $H$, for any $x \in H$, we have

$$
x=\sum_{j \in \mathcal{N}} \Lambda_{j}^{*} \widetilde{\Lambda}_{j} x .
$$

So for any $m \in \mathcal{N}$ and $g_{m} \in H_{m}$, we have

$$
\Lambda_{m}^{*} g_{m}=\sum_{j \in \mathcal{N}} \Lambda_{j}^{*} \widetilde{\Lambda}_{j}\left(\Lambda_{m}^{*} g_{m}\right)
$$

But $\Lambda_{m}^{*} g_{m}=\sum_{j \in \mathcal{N}} \Lambda_{j}^{*} \delta_{j, m} g_{j}$. So by [22, Lemma 2.1], we have

$$
\begin{gathered}
\left\|g_{m}\right\|^{2}=\sum_{j \in \mathcal{N}}\left\|\delta_{j, m} g_{j}\right\|^{2}=\sum_{j \in \mathcal{N}}\left\|\widetilde{\Lambda}_{j} \Lambda_{m}^{*} g_{m}\right\|^{2}+\sum_{j \in \mathcal{N}}\left\|\widetilde{\Lambda}_{j} \Lambda_{m}^{*} g_{m}-\delta_{j, m} g_{j}\right\|^{2} \\
=\left\|\widetilde{\Lambda}_{m} \Lambda_{m}^{*} g_{m}\right\|^{2}+\sum_{j \neq m}\left\|\widetilde{\Lambda}_{j} \Lambda_{m}^{*} g_{m}\right\|^{2}+\left\|g_{m}-\widetilde{\Lambda}_{m} \Lambda_{m}^{*} g_{m}\right\|^{2}+\sum_{j \neq m}\left\|\widetilde{\Lambda}_{j} \Lambda_{m}^{*} g_{m}\right\|^{2} .
\end{gathered}
$$

So $\sum_{j \neq m}\left\|\widetilde{\Lambda}_{j} \Lambda_{m}^{*} g_{m}\right\|^{2}=\frac{1}{2}\left[\left\|g_{m}\right\|^{2}-\left\|\widetilde{\Lambda}_{m} \Lambda_{m}^{*} g_{m}\right\|^{2}-\left\|g_{m}-\widetilde{\Lambda}_{m} \Lambda_{m}^{*} g_{m}\right\|^{2}\right]$.

(2) If $\widetilde{\Lambda}_{m} \Lambda_{m}^{*}=I_{H_{m}}$, from (1), we have that $\left\|\widetilde{\Lambda}_{j} \Lambda_{m}^{*} g_{m}\right\|^{2}=0$ for any $j \neq m$ and any $g_{m} \in H_{m}$. It implies that $\widetilde{\Lambda}_{j} \Lambda_{m}^{*}=0$ for any $j \neq m$. The second statement is obvious.

(3) If $\operatorname{ker}\left(I_{H_{m}}-\widetilde{\Lambda}_{m} \Lambda_{m}^{*}\right) \neq 0$, then there exists $g_{m} \in H_{m}$ with $g_{m} \neq 0$ such that $g_{m}=\widetilde{\Lambda}_{m} \Lambda_{m}^{*} g_{m}$. Then from (1), we have that $\sum_{j \neq m}\left\|\widetilde{\Lambda}_{j} \Lambda_{m}^{*} g_{m}\right\|^{2}=0$, which 
implies that $\widetilde{\Lambda}_{j} \Lambda_{m}^{*} g_{m}=0$ for any $j \neq m$. So $\left\langle\Lambda_{m}^{*} g_{m}, \widetilde{\Lambda}_{j}^{*} g_{j}\right\rangle=0$ for any $j \neq m$ and any $g_{j} \in H_{j}$. It follows that $\Lambda_{m}^{*} g_{m} \perp \overline{\operatorname{span}}\left\{\widetilde{\Lambda}_{j}^{*}\left(H_{j}\right)\right\}_{j \neq m}$. But $\widetilde{\Lambda}_{m} \Lambda_{m}^{*} g_{m}=g_{m} \neq 0$, so $\Lambda_{m}^{*} g_{m} \neq 0$. So $\overline{\operatorname{span}}\left\{\widetilde{\Lambda}_{j}^{*}\left(H_{j}\right)\right\}_{j \neq m} \neq H$, i.e., $\left\{\widetilde{\Lambda}_{j}\right\}_{j \neq m}$ is not $g$-complete. Since $\widetilde{\Lambda}_{j}=\Lambda_{j} S^{-1}$, it is easy to see that $\left\{\Lambda_{j}\right\}_{j \neq m}$ is not $g$-complete as well. Since

$$
\overline{\operatorname{range}}\left(I_{H_{m}}-\widetilde{\Lambda}_{m} \Lambda_{m}^{*}\right)=\operatorname{ker}\left(I_{H_{m}}-\Lambda_{m} \widetilde{\Lambda}_{m}^{*}\right)^{\perp}
$$

we have that

$$
\overline{\operatorname{range}}\left(I_{H_{m}}-\widetilde{\Lambda}_{m} \Lambda_{m}^{*}\right) \neq H_{m} \Leftrightarrow \operatorname{ker}\left(I_{H_{m}}-\Lambda_{m} \widetilde{\Lambda}_{m}^{*}\right) \neq 0 .
$$

Since $\left\{\Lambda_{j}: j \in \mathcal{N}\right\}$ and $\left\{\widetilde{\Lambda}_{j}: j \in \mathcal{N}\right\}$ are canonical dual $g$-frames for each other, by interchanging $\Lambda_{j}$ with $\widetilde{\Lambda}_{j}$, the second case can be proved similarly.

(4) Suppose that $I_{H_{m}}-\widetilde{\Lambda}_{m} \Lambda_{m}^{*}$ is surjective, then there exists an operator $T \in$ $B\left(H_{m}, H_{m}\right)$ such that $\left(I_{H_{m}}-\widetilde{\Lambda}_{m} \Lambda_{m}^{*}\right) T=I_{H_{m}}$. It follows that $T^{*}\left(I_{H_{m}}-\Lambda_{m} \widetilde{\Lambda}_{m}^{*}\right)=$ $I_{H_{m}}$. Since for any $f \in H$, we have $f=\sum_{j \in \mathcal{N}} \widetilde{\Lambda}_{j}^{*} \Lambda_{j} f$, so for any $m \in \mathcal{N}$,

$$
\begin{aligned}
& \Lambda_{m} f=\Lambda_{m} \sum_{j \in \mathcal{N}} \widetilde{\Lambda}_{j}^{*} \Lambda_{j} f=\sum_{j \in \mathcal{N}} \Lambda_{m} \widetilde{\Lambda}_{j}^{*} \Lambda_{j} f \\
& =\sum_{j \neq m} \Lambda_{m} \widetilde{\Lambda}_{j}^{*} \Lambda_{j} f+\Lambda_{m} \widetilde{\Lambda}_{m}^{*} \Lambda_{m} f
\end{aligned}
$$

It follows that $\left(I_{H_{m}}-\Lambda_{m} \widetilde{\Lambda}_{m}^{*}\right) \Lambda_{m} f=\sum_{j \neq m} \Lambda_{m} \widetilde{\Lambda}_{j}^{*} \Lambda_{j} f$. Since

$$
\begin{aligned}
& \left\|\sum_{j \neq m} \Lambda_{m} \widetilde{\Lambda}_{j}^{*} \Lambda_{j} f\right\|^{2}=\sup _{\|g\|=1}\left|\left\langle\sum_{j \neq m} \Lambda_{m} \widetilde{\Lambda}_{j}^{*} \Lambda_{j} f, g\right\rangle\right|^{2} \\
& =\sup _{\|g\|=1}\left|\sum_{j \neq m}\left\langle\Lambda_{j} f, \widetilde{\Lambda}_{j} \Lambda_{m}^{*} g\right\rangle\right|^{2} \leq \sum_{j \neq m}\left\|\Lambda_{j} f\right\|^{2} \cdot \sup _{\|g\|=1} \sum_{j \neq m}\left\|\widetilde{\Lambda}_{j} \Lambda_{m}^{*} g\right\|^{2} \\
& \leq \sum_{j \neq m}\left\|\Lambda_{j} f\right\|^{2} \cdot \sup _{\|g\|=1} \sum_{j \in \mathcal{N}}\left\|\widetilde{\Lambda}_{j} \Lambda_{m}^{*} g\right\|^{2} \leq \sum_{j \neq m}\left\|\Lambda_{j} f\right\|^{2} \cdot \frac{1}{A}\left\|\Lambda_{m}^{*} g\right\|^{2} \\
& \leq \sum_{j \neq m}\left\|\Lambda_{j} f\right\|^{2} \cdot \frac{1}{A}\left\|\Lambda_{m}^{*}\right\|^{2}
\end{aligned}
$$

so we have that

$$
\begin{aligned}
& \left\|\Lambda_{m} f\right\|^{2}=\left\|T^{*}\left(I_{H_{m}}-\Lambda_{m} \widetilde{\Lambda}_{m}^{*}\right) \Lambda_{m} f\right\|^{2} \\
& \leq\left\|T^{*}\right\|^{2} \cdot\left\|\left(I_{H_{m}}-\Lambda_{m} \widetilde{\Lambda}_{m}^{*}\right) \Lambda_{m} f\right\|^{2} \\
& =\left\|T^{*}\right\|^{2} \cdot\left\|\sum_{j \neq m} \Lambda_{m} \widetilde{\Lambda}_{j}^{*} \Lambda_{j} f\right\|^{2} \\
& \leq\left\|T^{*}\right\|^{2} \cdot \frac{1}{A} \cdot\left\|\Lambda_{m}^{*}\right\|^{2} \cdot \sum_{j \neq m}\left\|\Lambda_{j} f\right\|^{2} .
\end{aligned}
$$


Hence

$$
\begin{aligned}
& \sum_{j \in \mathcal{N}}\left\|\Lambda_{j} f\right\|^{2}=\left\|\Lambda_{m} f\right\|^{2}+\sum_{j \neq m}\left\|\Lambda_{j} f\right\|^{2} \\
& \leq\left(1+\left\|T^{*}\right\|^{2} \cdot \frac{1}{A} \cdot\left\|\Lambda_{m}^{*}\right\|^{2}\right) \cdot \sum_{j \neq m} \Lambda_{j} f\left\|^{2}=C \cdot \sum_{j \neq m} \Lambda_{j} f\right\|^{2},
\end{aligned}
$$

where $C=1+\left\|T^{*}\right\|^{2} \cdot \frac{1}{A} \cdot\left\|\Lambda_{m}^{*}\right\|^{2}$. Therefore

$$
\frac{A}{C}\|f\|^{2} \leq \frac{1}{C} \sum_{j \in \mathcal{N}}\left\|\Lambda_{j} f\right\|^{2} \leq \sum_{j \neq m}\left\|\Lambda_{j} f\right\|^{2} \leq \sum_{j \in \mathcal{N}}\left\|\Lambda_{j} f\right\|^{2} \leq B\|f\|^{2} .
$$

It implies that $\left\{\Lambda_{j}\right\}_{j \neq m}$ is a $g$-frame with frame bounds $\frac{A}{C}$ and $B$. For the second case, by the duality mentioned in (3), by interchanging $\Lambda_{j}$ with $\widetilde{\Lambda}_{j}$, we can proved similarly that $\left\{\widetilde{\Lambda}_{j}\right\}_{j \neq m}$ is a $g$-frame for $H$. Since $\left\{\Lambda_{j}\right\}_{j \neq m}$ is similar to $\left\{\widetilde{\Lambda}_{j}\right\}_{j \neq m}$, it is easy to see that $\left\{\Lambda_{j}\right\}_{j \neq m}$ is a $g$-frame for $H$.

Note that when $\operatorname{dim} H_{j}<\infty$ for all $j \in \mathcal{N}$, then $\operatorname{ker}\left(I_{H_{m}}-\widetilde{\Lambda}_{m} \Lambda_{m}^{*}\right)=0 \Leftrightarrow$ $\operatorname{range}\left(I_{H_{m}}-\widetilde{\Lambda}_{m} \Lambda_{m}^{*}\right)=\overline{\operatorname{range}}\left(I_{H_{m}}-\widetilde{\Lambda}_{m} \Lambda_{m}^{*}\right)=H$. The following result is obtained directly from Lemma 2.11 (3) and (4), which was also proved in [18] differently. It characterizes the exact $g$-frames under the constraint that $\operatorname{dim} H_{j}<\infty$ for all $j \in \mathcal{N}$.

Corollary 2.12. Let $\Lambda_{j} \in B\left(H, H_{j}\right)$, and $\operatorname{dim} H_{j}<\infty$ for $j \in \mathcal{N}$. Then the following statements are equivalent:

(1) The sequence $\left\{\Lambda_{j}: j \in \mathcal{N}\right\}$ is an exact $g$-frame for $H$ with respect to $\left\{H_{j}\right.$ : $j \in \mathcal{N}\}$.

(2) The sequence $\left\{\Lambda_{j}: j \in \mathcal{N}\right\}$ is a $g$-frame for $H$ with respect to $\left\{H_{j}: j \in \mathcal{N}\right\}$ and $\left\{\Lambda_{j}: j \in \mathcal{N}, j \neq j_{0}\right\}$ is not g-complete for every $j_{0} \in \mathcal{N}$.

The following lemma is from the same Chinese journal as that of Lemma 2.11, which applies the results of Lemma 2.11 to study the redundancy related properties of normalized tight $g$-frames. We include its proof for convenience.

Lemma 2.13. [9] Suppose $\left\{\Lambda_{j}: j \in \mathcal{N}\right\}$ is a normalized tight g-frame for $H$. Then for any $m \in \mathcal{N}$, if $\operatorname{ker}\left(I_{H_{m}}-\Lambda_{m} \Lambda_{m}^{*}\right) \neq\{0\}$, then $\left\{\Lambda_{j}\right\}_{j \neq m}$ is not g-complete. If $\operatorname{ker}\left(I-\Lambda_{m}^{*} \Lambda_{m}\right)=\{0\}$, then $\left\{\Lambda_{j}\right\}_{j \neq m}$ is a g-frame for $H$.

Proof. Since $\left\{\Lambda_{j}: j \in \mathcal{N}\right\}$ is a normalized tight $g$-frame for $H$, the frame operator associated with $\left\{\Lambda_{j}: j \in \mathcal{N}\right\}$ is $S=I$. It implies that the canonical dual $g$-frame of $\left\{\Lambda_{j}: j \in \mathcal{N}\right\}$ is $\left\{\Lambda_{j} S^{-1}: j \in \mathcal{N}\right\}=\left\{\Lambda_{j}: j \in \mathcal{N}\right\}$. For any $m \in \mathcal{N}$, if $\operatorname{ker}\left(I_{H_{m}}-\Lambda_{m} \Lambda_{m}^{*}\right) \neq\{0\}$, then $\left\{\Lambda_{j}\right\}_{j \neq m}$ is not $g$-complete by Lemma 2.11 (3). Since $f=S f=\sum_{j \in \mathcal{N}} \Lambda_{j}^{*} \Lambda_{j} f$ for any $f \in H, \sum_{j \in \mathcal{N}} \Lambda_{j}^{*} \Lambda_{j}=I$, which implies that $\Lambda_{m}^{*} \Lambda_{m} \leq I$. If $\operatorname{ker}\left(I-\Lambda_{m}^{*} \Lambda_{m}\right)=\{0\}$, then

$$
\begin{aligned}
& \left\|\Lambda_{m}^{*} \Lambda_{m}\right\|=\sup _{\|x\|=1}\left\langle\Lambda_{m}^{*} \Lambda_{m} x, x\right\rangle \\
& =\left\langle\Lambda_{m}^{*} \Lambda_{m} x_{0}, x_{0}\right\rangle<\left\langle x_{0}, x_{0}\right\rangle=1,
\end{aligned}
$$

where $x_{0} \in\{x \in H:\|x\|=1\}$. Hence $\left\|\Lambda_{m} \Lambda_{m}^{*}\right\|=\left\|\Lambda_{m}^{*} \Lambda_{m}\right\|<1$. It implies that $I_{H_{m}}-\Lambda_{m} \Lambda_{m}^{*}$ is invertible. So $\left\{\Lambda_{j}\right\}_{j \neq m}$ is a $g$-frame by Lemma 2.11 (4). 
The following lemma shows that the condition for a sequence of operators to be a $g$-Riesz basis is stronger than that for it to be an exact $g$-frame.

Lemma 2.14. [22] A g-Riesz basis $\left\{\Lambda_{j}: j \in \mathcal{N}\right\}$ is an exact $g$-frame. Moreover, it is g-biorthonormal with its dual $\left\{\widetilde{\Lambda}_{j}: j \in \mathcal{N}\right\}$.

The following lemma shows that when $\operatorname{dim} H_{j}<\infty$ for any $j \in \mathcal{N}$, any finite sequence of operators is a $g$-frame for its generating subspace. Since it is from a Chinese journal, we include its detailed proof for the sake of convenience.

Lemma 2.15. [10] Suppose $\operatorname{dim} H_{j}<\infty, \Lambda_{j} \in B\left(H, H_{j}\right)$. Then for any $n \in \mathcal{N}$, $\left\{\Lambda_{j}\right\}_{j=1}^{n}$ is a g-frame for $\overline{\operatorname{span}}\left\{\Lambda_{j}^{*}\left(H_{j}\right)\right\}_{j=1}^{n}$.

Proof. For any $f \in \overline{\operatorname{span}}\left\{\Lambda_{j}^{*}\left(H_{j}\right)\right\}_{j=1}^{n}$, we have

$$
\sum_{j=1}^{n}\left\|\Lambda_{j} f\right\|^{2} \leq \sum_{j=1}^{n}\left\|\Lambda_{j}\right\|^{2} \cdot\|f\|^{2}=B\|f\|^{2}
$$

where $B=\sum_{j=1}^{n}\left\|\Lambda_{j}\right\|^{2}$. So $\left\{\Lambda_{j}\right\}_{j=1}^{n}$ is a $g$-Bessel sequence with bound $B$ for $\overline{\operatorname{span}}\left\{\Lambda_{j}^{*}\left(H_{j}\right)\right\}_{j=1}^{n}$. Next, we aim to show the existence of lower frame bound. Let

$$
\phi(f)=\sum_{j=1}^{n}\left\|\Lambda_{j} f\right\|^{2}, \forall f \in \overline{\operatorname{span}}\left\{\Lambda_{j}^{*}\left(H_{j}\right)\right\}_{j=1}^{n},
$$

then $\phi$ is a continuous function on $\overline{\operatorname{span}}\left\{\Lambda_{j}^{*}\left(H_{j}\right)\right\}_{j=1}^{n}$. Since $\overline{\operatorname{span}}\left\{\Lambda_{j}^{*}\left(H_{j}\right)\right\}_{j=1}^{n}$ is of finite dimensional, its unit circle is a compact set. Hence there exists $y \in$ $\overline{\operatorname{span}}\left\{\Lambda_{j}^{*}\left(H_{j}\right)\right\}_{j=1}^{n}$ with $\|y\|=1$ such that

$$
\phi(y)=\sum_{j=1}^{n}\left\|\Lambda_{j} y\right\|^{2}=\inf \left\{\sum_{j=1}^{n}\left\|\Lambda_{j} f\right\|^{2} \mid f \in \overline{\operatorname{span}}\left\{\Lambda_{j}^{*}\left(H_{j}\right)\right\}_{j=1}^{n},\|f\|=1\right\} .
$$

Let $A=\phi(y)$, then $A \geq 0$. But if $A=0$, then $\Lambda_{j} y=0$ for $j=1, \cdots, n$. Thus

$$
\left\langle y, \Lambda_{j}^{*}\left(g_{j}\right)\right\rangle=\left\langle\Lambda_{j} y, g_{j}\right\rangle=0, \forall g_{j} \in H_{j}, j=1, \cdots, n .
$$

It implies that $y \perp \overline{\operatorname{span}}\left\{\Lambda_{j}^{*}\left(H_{j}\right)\right\}_{j=1}^{n}$. So $y=0$, which contradicts with the fact that $\|y\|=1$. Therefore $A>0$. Since for any $f \in \overline{\operatorname{span}}\left\{\Lambda_{j}^{*}\left(H_{j}\right)\right\}_{j=1}^{n}$ and $f \neq 0$, we have

$$
\begin{aligned}
& \sum_{j=1}^{n}\left\|\Lambda_{j} f\right\|^{2}=\sum_{j=1}^{n} \frac{\left\|\Lambda_{j} f\right\|^{2}}{\|f\|^{2}} \cdot\|f\|^{2} \\
& =\|f\|^{2} \cdot \sum_{j=1}^{n}\left\|\Lambda_{j}\left(\frac{f}{\|f\|}\right)\right\|^{2} \geq A \cdot\|f\|^{2} .
\end{aligned}
$$

Hence $\left\{\Lambda_{j}\right\}_{j=1}^{n}$ is a $g$-frame for $\overline{\operatorname{span}}\left\{\Lambda_{j}^{*}\left(H_{j}\right)\right\}_{j=1}^{n}$ with fame bounds $A$ and $B$.

The following lemma characterizes $g$-Riesz bases from two different viewpoints.

Lemma 2.16. [11] Let $\Lambda_{j} \in B\left(H, H_{j}\right), j \in \mathcal{N}$. Then the following statements are equivalent:

(1) The sequence $\left\{\Lambda_{j}\right\}_{j \in \mathcal{N}}$ is a g-Riesz basis for $H$ with respect to $\left\{H_{j}\right\}_{j \in \mathcal{N}}$. 
(2) The sequence $\left\{\Lambda_{j}\right\}_{j \in \mathcal{N}}$ is a g-frame for $H$ with respect to $\left\{H_{j}\right\}_{j \in \mathcal{N}}$ and $\left\{\Lambda_{j}\right\}_{j \in \mathcal{N}}$ is g-linearly independent.

(3) The sequence $\left\{\Lambda_{j}\right\}_{j \in \mathcal{N}}$ is a g-basis and a g-frame with respect to $\left\{H_{j}\right\}_{j \in \mathcal{N}}$.

\section{REDUNDANCY OF $g$-FRAMES}

In this section, we study the redundancy of $g$-frames in Hilbert spaces.

The following is one of our main results which characterizes $g$-frames without any redundancy, i.e., $g$-Riesz bases.

Theorem 3.1. Let $\left\{\Lambda_{i} \in B\left(H, H_{i}\right): i \in \mathcal{N}\right\}$ be a g-frame for $H$ with $A$ as its lower frame bound and $B$ as its upper frame bound. Then the following statements are equivalent.

(1). $\left\{\Lambda_{i}: i \in \mathcal{N}\right\}$ is a $g$-Riesz basis for $H$.

(2). $\left\{\Lambda_{i}: i \in \mathcal{N}\right\}$ and $\left\{\widetilde{\Lambda}_{i}: i \in \mathcal{N}\right\}$ are g-biorthonormal.

(3). There exists a sequence $\left\{\Gamma_{i} \in B\left(H, H_{i}\right): i \in \mathcal{N}\right\}$ which is g-biorthonormal with $\left\{\Lambda_{i}: i \in \mathcal{N}\right\}$.

(4). $\left\{\Lambda_{i}: i \in \mathcal{N}\right\}$ is g-minimal.

(5). $\left\{\Lambda_{i}: i \in \mathcal{N}\right\}$ is g-independent.

(6). $\left\{\Lambda_{i}: i \in \mathcal{N}\right\}$ is a g-basis for $H$.

In case that $\operatorname{dim} H_{j}<\infty$ for any $j \in \mathcal{N}$. Let $A_{n}$ denote the optimal lower frame bound for $g$-frame sequence $\left\{\Lambda_{j}\right\}_{j=1}^{n}$. Then the above statements are also equivalent to the following two statements.

(7). $\left\{\Lambda_{i}: i \in \mathcal{N}\right\}$ is finitely g-linearly independent and in $f_{k \in \mathcal{N}} A_{k}>0$.

(8). $\left\{\Lambda_{i}: i \in \mathcal{N}\right\}$ is finitely $g$-linearly independent and $\lim _{n \rightarrow \infty} A_{n}>0$.

Proof. $(1) \Rightarrow(2)$ : This implication is already proved in [22], here we include a different argument which is more directly. Since $\left\{\Lambda_{i}: i \in \mathcal{N}\right\}$ is a $g$-Riesz basis for $H$, there exists a $g$-orthonormal basis $\left\{Q_{i}: i \in \mathcal{N}\right\}$ and a bounded invertible operator $T \in B(H)$ such that $\Lambda_{j}=Q_{j} T$ for any $j \in \mathcal{N}$ by Lemma 2.10. Let $S$ denote the frame operator associated with $\left\{\Lambda_{j}: j \in \mathcal{N}\right\}$. Then $S f=$ $\sum_{j \in \mathcal{N}} \Lambda_{j}^{*} \Lambda_{j} f, \forall f \in H$. So $S f=\sum_{j \in \mathcal{N}} T^{*} Q_{j}^{*} Q_{j} T f, \forall f \in H$. So $\left(T^{*}\right)^{-1} S f=$ $\sum_{j \in \mathcal{N}} Q_{j}^{*} Q_{j} T f, \forall f \in H$. It follows that $\left(T^{*}\right)^{-1} S T^{-1}=\sum_{j \in \mathcal{N}} Q_{j}^{*} Q_{j}=I$. Hence $S=T^{*} T$. So $\widetilde{\Lambda}_{j}=\Lambda_{j} S^{-1}=\Lambda_{j} T^{-1}\left(T^{*}\right)^{-1}, \forall j \in \mathcal{N}$. Then

$$
\widetilde{\Lambda}_{j} \Lambda_{j}^{*}=\Lambda_{j} T^{-1}\left(T^{*}\right)^{-1} T^{*} Q_{j}^{*}=\Lambda_{j} T^{-1} Q_{j}^{*}=Q_{j} Q_{j}^{*}=I_{H_{j}}, \forall j \in \mathcal{N} .
$$

So $\left\{\widetilde{\Lambda}_{j}: j \in \mathcal{N}\right\}$ is $g$-biorthonormal with $\left\{\Lambda_{j}: j \in \mathcal{N}\right\}$ by Lemma $2.11(2)$.

$(2) \Rightarrow(3)$ is obvious.

$(3) \Rightarrow(4):$ Given $m \in \mathcal{N}, g_{m} \in H_{m}$ with $g_{m} \neq 0$, since $\left\{\Gamma_{j}: j \in \mathcal{N}\right\}$ is $g$-biorthogonal with $\left\{\Lambda_{j}: j \in \mathcal{N}\right\}$, for any $j \in \mathcal{N}$ with $j \neq m, g_{j} \in H_{j}$, we have $\left\langle\Gamma_{m}^{*} g_{m}, \Lambda_{j}^{*} g_{j}\right\rangle=0$. So $\Gamma_{m}^{*} g_{m} \perp \overline{\operatorname{span}}_{j \neq m}\left\{\Lambda_{j}^{*} g_{j}: g_{j} \in H_{j}\right\}$. But $\left\langle\Gamma_{m}^{*} g_{m}, \Lambda_{m}^{*} g_{m}\right\rangle=\left\|g_{m}\right\|^{2} \neq 0$. It follows that $\Lambda_{m}^{*} g_{m} \notin \overline{\operatorname{span}}_{j \neq m}\left\{\Lambda_{j}^{*} g_{j}: g_{j} \in H_{j}\right\}$, so $\left\{\Lambda_{j}: j \in \mathcal{N}\right\}$ is $g$-minimal.

$(4) \Rightarrow(5)$ : Suppose that $\sum_{j \in \mathcal{N}} \Lambda_{j}^{*} g_{j}=0$. If there exists $k \in \mathcal{N}$ such that $g_{k} \neq 0$, then $\Lambda_{k}^{*} g_{k}=-\sum_{j \in \mathcal{N}, j \neq k} \Lambda_{j}^{*} g_{j}$. So $\Lambda_{k}^{*} g_{k} \in \overline{\operatorname{span}}_{j \neq k}\left\{\Lambda_{j}^{*} g_{j}: g_{j} \in H_{j}\right\}$, which contradicts with the fact that $\left\{\Lambda_{j}: j \in \mathcal{N}\right\}$ is $g$-minimal.

$(5) \Rightarrow(6)$ : Since $\left\{\Lambda_{j}: j \in \mathcal{N}\right\}$ is a $g$-frame, for every vector $f \in H$, there exists 
a sequence $\left\{g_{j} \in H_{j}: j \in \mathcal{N}\right\}$ such that $f=\sum_{j \in \mathcal{N}} \Lambda_{j}^{*} g_{j}$. Since $\left\{\Lambda_{j}: j \in \mathcal{N}\right\}$ is $g$-linearly independent, the representation is unique. So $\left\{\Lambda_{j}: j \in \mathcal{N}\right\}$ is a $g$-basis for $H$.

$(6) \Rightarrow(1)$ : By Lemma 2.16, it is true.

In case that $\operatorname{dim}_{j}<\infty$ for any $j \in \mathcal{N}$. We will show that $(6) \Rightarrow(7) \Rightarrow(8) \Rightarrow$ (1). Since $(1) \Rightarrow(6)$ has already been shown, all the statements are equivalent as required.

$(6) \Rightarrow(7)$ : Since $\left\{\Lambda_{j}: j \in \mathcal{N}\right\}$ is a $g$-basis for $H,\left\{\Lambda_{j}: j \in \mathcal{N}\right\}$ is finitely $g$-linearly independent. Since $\left\{\Lambda_{j}: j \in \mathcal{N}\right\}$ is a $g$-basis and a $g$-frame for $H$, $\left\{\Lambda_{j}: j \in \mathcal{N}\right\}$ is a $g$-Riesz basis for $H$. So for any $n \in \mathcal{N},\left\{\Lambda_{j}\right\}_{j=1}^{n}$ is a $g$-Riesz sequence by Lemma 2.15. Since the optimal lower frame bound of $\left\{\Lambda_{j}\right\}_{j=1}^{n}$ is $A_{n}$, we have $A_{n} \geq A$. Hence $i n f_{k \in \mathcal{N}} A_{k}>0$.

$(7) \Rightarrow(8)$ : It suffices to show that $\lim _{n \rightarrow \infty} A_{n}>0$. Since $A_{n}$ is the optimal lower frame bound for $\left\{\Lambda_{j}\right\}_{j=1}^{n}$, we have that $A_{n} \geq A_{n+1} \geq i n f_{k \in \mathcal{N}} A_{k}>0$. So $\lim _{n \rightarrow \infty} A_{n}$ exists and $\lim _{n \rightarrow \infty} A_{n}=i n f_{k \in \mathcal{N}} A_{k}>0$.

$(8) \Rightarrow(1)$ : Since $\left\{\Lambda_{j}\right\}_{j=1}^{n}$ is $g$-linearly independent for any $n \in \mathcal{N}$, hence $\left\{\Lambda_{j}\right\}_{j=1}^{n}$ is a $g$-Riesz basis for $\overline{\operatorname{span}}\left\{\Lambda_{j}^{*} g_{j}: g_{j} \in H_{j}, j=1, \cdots, n\right\}$ by Lemma 2.15 and $A_{0}=i n f_{k \in \mathcal{N}} A_{k}=\lim _{n \rightarrow \infty} A_{n}>0$ is a lower frame bound for $\left\{\Lambda_{j}\right\}_{j=1}^{n}$. So for any $\left\{g_{j}\right\}_{j=1}^{n}$ we have $A_{0} \sum_{j=1}^{n}\left\|g_{j}\right\|^{2} \leq\left\|\sum_{j=1}^{n} \Lambda_{j}^{*} g_{j}\right\|^{2}$. Let $B$ be the upper frame bound for $\left\{\Lambda_{j}: j \in \mathcal{N}\right\}$. Then $\left\|\sum_{j=1}^{n} \Lambda_{j}^{*} g_{j}\right\|^{2} \leq B \sum_{j=1}^{n}\left\|g_{j}\right\|^{2}$. So $A_{0} \sum_{j=1}^{n}\left\|g_{j}\right\|^{2} \leq$ $\left\|\sum_{j=1}^{n} \Lambda_{j}^{*} g_{j}\right\|^{2} \leq B \sum_{j=1}^{n}\left\|g_{j}\right\|^{2}$ for any finite set $\left\{g_{j}: g_{j} \in H_{j}, j=1, \cdots, n\right\}$. Since $\left\{\Lambda_{j}: j \in \mathcal{N}\right\}$ is a $g$-frame, $\left\{\Lambda_{j}: j \in \mathcal{N}\right\}$ is $g$-complete. It follows that $\left\{\Lambda_{j}: j \in \mathcal{N}\right\}$ is a $g$-Riesz basis for $H$.

The following result gives a necessary condition for a normalized tight $g$-frame to be exact.

Theorem 3.2. Suppose that $\left\{\Lambda_{j}: j \in \mathcal{N}\right\}$ is a normalized tight g-frame for $H$. If $\left\{\Lambda_{j}: j \in \mathcal{N}\right\}$ is an exact $g$-frame then $\left\|\Lambda_{j}\right\|=1$ for $\forall j \in \mathcal{N}$.

Proof. Since $\left\{\Lambda_{j}: j \in \mathcal{N}\right\}$ is a normalized tight $g$-frame for $H, \sum_{j \in \mathcal{N}}\left\|\Lambda_{j} f\right\|^{2}=$ $\|f\|^{2}$ for any $f \in H$. So $\left\|\Lambda_{j} f\right\| \leq\|f\|$ for any $j \in \mathcal{N}$ and any $f \in H$. It follows that $\left\|\Lambda_{j}\right\| \leq 1$ for any $j \in \mathcal{N}$. If there exists some $k \in \mathcal{N}$ such that $\left\|\Lambda_{k}\right\|<1$, then $\left\|\Lambda_{k}^{*} \Lambda_{k}\right\|<1$. So $I-\Lambda_{k}^{*} \Lambda_{k}$ is invertible. Hence $\left\{\Lambda_{j}: j \in \mathcal{N}, j \neq k\right\}$ is a $g$-frame by Lemma 2.13, which contradicts with the fact that $\left\{\Lambda_{j}: j \in \mathcal{N}\right\}$ is exact. So $\left\|\Lambda_{j}\right\|=1$ for all $j \in \mathcal{N}$.

As an application of Theorem 3.2, we give a complete characterization for a normalized tight frame to be exact as follows. This result is already shown in [3], but here we prove it by the viewpoint of $g$-frames.

Corollary 3.3. Suppose that $\left\{f_{j}: j \in \mathcal{N}\right\}$ is a normalized tight frame for $H$. Then $\left\{f_{j}: j \in \mathcal{N}\right\}$ is an exact frame if and only if $\left\{f_{j}: j \in \mathcal{N}\right\}$ is an orthonormal basis for $H$.

Proof. The sufficient condition is obvious. Now we move to prove the necessary condition. Suppose that $\left\{f_{j}: j \in \mathcal{N}\right\}$ is an exact frame. Let $\Lambda_{f_{j}}(f)=\left\langle f, f_{j}\right\rangle$ 
for each $f \in H$ and each $j \in \mathcal{N}$. Then $\sum_{j \in \mathcal{N}}\left\|\Lambda_{f_{j}} f\right\|^{2}=\sum_{j \in \mathcal{N}}\left|\left\langle f, f_{j}\right\rangle\right|^{2}$. So $\left\{f_{j}: j \in \mathcal{N}\right\}$ is an exact frame for $H$ if and only if $\left\{\Lambda_{f_{j}}: j \in \mathcal{N}\right\}$ is an exact $g$-frame for $H$ with respect to $\left\{H_{j}: j \in \mathcal{N}\right\}$ where $H_{j}=\mathcal{C}$ for all $j \in \mathcal{N}$. So $\left\{\Lambda_{f_{j}}: j \in \mathcal{N}\right\}$ is an exact $g$-frame for $H$. By Theorem 3.2 we know that $\left\|\Lambda_{f_{j}}\right\|=1$ for each $j \in \mathcal{N}$. Since $\left\|\Lambda_{f_{j}}\right\|=\left\|f_{j}\right\|$ for each $j \in \mathcal{N}$, therefore $\left\|f_{j}\right\|=1$ for each $j \in \mathcal{N}$. Since for any $f \in H$ we have that $\|f\|^{2}=\sum_{j \in \mathcal{N}}\left|\left\langle f, f_{j}\right\rangle\right|^{2}$, in particular, for any $k \in \mathcal{N}$ we have $\left\|f_{k}\right\|^{2}=\sum_{j \in \mathcal{N}}\left|\left\langle f_{k}, f_{j}\right\rangle\right|^{2}=\left|\left\langle f_{k}, f_{k}\right\rangle\right|^{2}+\sum_{j \neq k}\left|\left\langle f_{k}, f_{j}\right\rangle\right|^{2}$. It follows that $\left\langle f_{i}, f_{j}\right\rangle=\delta_{i, j}$. So $\left\{f_{j}: j \in \mathcal{N}\right\}$ is an orthonormal basis for $H$.

The following corollary gives a complete characterization for a normalized tight $g$-frame to be a $g$-orthonormal basis.

Corollary 3.4. Suppose that $\left\{\Lambda_{j}: j \in \mathcal{N}\right\}$ is a normalized tight g-frame for $H$. Then $\left\{\Lambda_{j}: j \in \mathcal{N}\right\}$ is a g-orthonormal basis for $H$ if and only if $\Lambda_{j}$ is a co-isometry for any $j \in \mathcal{N}$.

Proof. $\Rightarrow$ : This direction is straightforward and we can also find its proof in [20, Proposition 2.14].

$\Leftarrow$ : One proof for this direction is given in [17, Corollary 2.6], but here we give a new different proof by using Lemma 2.11. Since $\Lambda_{m}$ is a co-isometry for any $m \in \mathcal{N}$, hence $\Lambda_{m} \Lambda_{m}^{*}=I_{H_{m}}$ for each $m \in \mathcal{N}$. So $\left\{\Lambda_{j}: j \in \mathcal{N}\right\}$ is $g$-biothogonal with itself by Lemma 2.11(2), which means that $\left\langle\Lambda_{i}^{*} g_{i}, \Lambda_{j}^{*} g_{j}\right\rangle=\delta_{i, j}\left\langle g_{i}, g_{j}\right\rangle$ for any $i, j \in \mathcal{N}$ and any $g_{i} \in H_{i}, g_{j} \in H_{j}$. Since $\left\{\Lambda_{j}: j \in \mathcal{N}\right\}$ is a normalized tight $g$-frame for $H$, we have that $\|f\|^{2}=\sum_{j \in \mathcal{N}}\left\|\Lambda_{j} f\right\|^{2}$. So $\left\{\Lambda_{j}: j \in \mathcal{N}\right\}$ is a $g$-orthonormal basis for $H$.

The following result is one of our main results which gives a complete characterization such that the class of $g$-Riesz bases is the same as the class of exact $g$-frames under the restriction that all $H_{j}$ are finite dimensional.

Theorem 3.5. Let $H, K$ be Hilbert spaces, $\left\{H_{j}: j \in \mathcal{N}\right\}$ be a sequence of closed subspaces of $K$ with $\operatorname{dim} H_{j}<\infty$ for any $j \in \mathcal{N}$. Then $\left\{\Lambda_{j}: j \in \mathcal{N}\right\}$ is an exact $g$-frame for $H$ with respect to $\left\{H_{j}: j \in \mathcal{N}\right\}$ is equivalent that $\left\{\Lambda_{j}: j \in \mathcal{N}\right\}$ is a $g$-Riesz basis for $H$ with respect to $\left\{H_{j}: j \in \mathcal{N}\right\}$ if and only if dim $H_{j}=1$ for any $j \in \mathcal{N}$.

Proof. $\Rightarrow$ : Suppose that $\left\{\Lambda_{j}: j \in \mathcal{N}\right\}$ is an exact $g$-frame for $H$ is equivalent that $\left\{\Lambda_{j}: j \in \mathcal{N}\right\}$ is a $g$-Riesz basis for $H$. If for any $m \in \mathcal{N}$, range $\left(I_{H_{m}}-\widetilde{\Lambda}_{m} \Lambda_{m}^{*}\right)=$ $\overline{\operatorname{range}}\left(I_{H_{m}}-\widetilde{\Lambda}_{m} \Lambda_{m}^{*}\right) \neq H_{m}$, then $\left\{\Lambda_{j}: j \in \mathcal{N}, j \neq m\right\}$ is not $g$-complete by Lemma $2.11(3)$, hence $\left\{\Lambda_{j}: j \in \mathcal{N}\right\}$ is an exact $g$-frame. It implies that $\left\{\Lambda_{j}\right.$ : $j \in \mathcal{N}\}$ is a $g$-Riesz basis. It follows that $\left\{\Lambda_{j}: j \in \mathcal{N}\right\}$ is $g$-biorthogonal with $\left\{\widetilde{\Lambda}_{j}: j \in \mathcal{N}\right\}$ by Lemma 2.14. Hence for any $m \in \mathcal{N}$, we have that $\widetilde{\Lambda}_{m} \Lambda_{m}^{*}=I_{H_{m}}$ by Lemma $2.11(2)$. So $I_{H_{m}}-\widetilde{\Lambda}_{m} \Lambda_{m}^{*}=0$ for any $m \in \mathcal{N}$. So we get that range $\left(I_{H_{m}}-\widetilde{\Lambda}_{m} \Lambda_{m}^{*}\right) \neq H_{m}$ if and only if $I_{H_{m}}-\widetilde{\Lambda}_{m} \Lambda_{m}^{*}=0$ for any $m \in \mathcal{N}$. It follows that $\operatorname{dim} H_{j}=1$ for any $j \in \mathcal{N}$. In fact, if there exists $j_{0} \in \mathcal{N}$ such that $\operatorname{dim} H_{j_{0}}>1$, then $\operatorname{range}\left(I_{H_{j_{0}}}-\widetilde{\Lambda}_{j_{0}} \Lambda_{j_{0}}^{*}\right) \neq H_{j_{0}} \nRightarrow I_{H_{j_{0}}}-\widetilde{\Lambda}_{j_{0}} \Lambda_{j_{0}}^{*}=0$. $\Leftarrow$ : One proof of this direction was given by [18, Corollary 2.7]. Now we give a 
different new proof. Since $\operatorname{dim} H_{j}=1$ for any $j \in \mathcal{N}, \overline{\operatorname{range}}\left(I_{H_{m}}-\widetilde{\Lambda}_{m} \Lambda_{m}^{*}\right)=$ $\operatorname{range}\left(I_{H_{m}}-\widetilde{\Lambda}_{m} \Lambda_{m}^{*}\right)$. By the dimension formula, we have that

$$
1=\operatorname{dim}\left(\operatorname{range}\left(I_{H_{m}}-\widetilde{\Lambda}_{m} \Lambda_{m}^{*}\right)\right)+\operatorname{dim}\left(\operatorname{ker}\left(\left(I_{H_{m}}-\widetilde{\Lambda}_{m} \Lambda_{m}^{*}\right)\right) .\right.
$$

So $\operatorname{dim}\left(\operatorname{range}\left(I_{H_{m}}-\widetilde{\Lambda}_{m} \Lambda_{m}^{*}\right)\right)=0$ if and only if $\operatorname{dim}\left(\operatorname{ker}\left(I_{H_{m}}-\widetilde{\Lambda}_{m} \Lambda_{m}^{*}\right)\right)=1$ for any $m \in \mathcal{N}$. It follows that $\operatorname{ker}\left(I_{H_{m}}-\widetilde{\Lambda}_{m} \Lambda_{m}^{*}\right)=H_{m}$ if and only if $\operatorname{range}\left(I_{H_{m}}-\right.$ $\left.\widetilde{\Lambda}_{m} \Lambda_{m}^{*}\right)=0$ for any $m \in \mathcal{N}$. Since $\operatorname{dim} H_{m}=1$, range $\left(I_{H_{m}}-\widetilde{\Lambda}_{m} \Lambda_{m}^{*}\right)=0$ or $\operatorname{range}\left(I_{H_{m}}-\widetilde{\Lambda}_{m} \Lambda_{m}^{*}\right)=H_{m}$. Thus for any $m \in \mathcal{N}$, we have that $\widetilde{\Lambda}_{m} \Lambda_{m}^{*}=I_{H_{m}}$ if and only if range $\left(I_{H_{m}}-\widetilde{\Lambda}_{m} \Lambda_{m}^{*}\right) \neq H_{m}$. It implies that $\left\{\Lambda_{j}: j \in \mathcal{N}\right\}$ is an exact $g$-frame if and only if $\left\{\Lambda_{j}: j \in \mathcal{N}\right\}$ is a $g$-Riesz basis for $H$ by Lemma 2.11 (2) and (3).

In the last result of this section, we consider the extension of normalized tight $g$-frames preserving the normalization property.

Theorem 3.6. Suppose that $\left\{\Lambda_{j}: j \in \mathcal{N}\right\}$ is a normalized tight g-frame for $H$. Then

(1) $\Lambda_{m} \Lambda_{m}^{*} \leq I_{H_{m}}$ for any $m \in \mathcal{N}$.

(2) The only way to enlarge $\left\{\Lambda_{j}: j \in \mathcal{N}\right\}$ in such a way that it remains to be a normalized tight $g$-frame is to add zero operators.

Proof. (1) Since $\left\{\Lambda_{j}: j \in \mathcal{N}\right\}$ is a normalized tight $g$-frame for $H$, we have that $\|g\|^{2}=\sum_{j \in \mathcal{N}}\left\|\Lambda_{j} g\right\|^{2}$ for any $g \in H$. Thus for any $m \in \mathcal{N}$ and $f \in H_{m}$, we have

$$
\left\|\Lambda_{m}^{*} f\right\|^{2}=\sum_{j \in \mathcal{N}}\left\|\Lambda_{j} \Lambda_{m}^{*} f\right\|^{2}=\left\|\Lambda_{m} \Lambda_{m}^{*} f\right\|^{2}+\sum_{j \in \mathcal{N}, j \neq m}\left\|\Lambda_{j} \Lambda_{m}^{*} f\right\|^{2} .
$$

So $\left\|\Lambda_{m}^{*} f\right\|^{2} \geq\left\|\Lambda_{m} \Lambda_{m}^{*} f\right\|^{2}$, i.e.,

$$
\left\langle\Lambda_{m}^{*} f, \Lambda_{m}^{*} f\right\rangle \geq\left\langle\Lambda_{m} \Lambda_{m}^{*} f, \Lambda_{m} \Lambda_{m}^{*} f\right\rangle
$$

so $\left\langle f, \Lambda_{m} \Lambda_{m}^{*} f\right\rangle \geq\left\langle f,\left(\Lambda_{m} \Lambda_{m}^{*}\right)^{2} f\right\rangle$. Hence $\Lambda_{m} \Lambda_{m}^{*} \geq\left(\Lambda_{m} \Lambda_{m}^{*}\right)^{2}$. It follows that

$$
\Lambda_{m} \Lambda_{m}^{*}\left(I_{H_{m}}-\Lambda_{m} \Lambda_{m}^{*}\right) \geq 0
$$

Since $\Lambda_{m} \Lambda_{m}^{*} \geq 0, I_{H_{m}}-\Lambda_{m} \Lambda_{m}^{*} \geq 0$, i.e., $\Lambda_{m} \Lambda_{m}^{*} \leq I_{H_{m}}, \forall m \in \mathcal{N}$.

(2). Suppose $\left\{\Lambda_{j}: j \in \Delta\right\}$ is a subset of $\left\{\Lambda_{j}: j \in \mathcal{N}\right\}$ and it is also a normalized tight $g$-frame for $H$. If $j \notin \Delta$ and $f \in H_{j}$, then

$$
\left\|\Lambda_{j}^{*} f\right\|^{2}=\sum_{i \in \mathcal{N}}\left\|\Lambda_{i} \Lambda_{j}^{*} f\right\|^{2}=\sum_{k \in \Delta}\left\|\Lambda_{k} \Lambda_{j}^{*} f\right\|^{2}
$$

Thus $\sum_{i \notin \Delta}\left\|\Lambda_{i} \Lambda_{j}^{*}\right\|^{2}=0, \forall f \in H$. So $\Lambda_{i} \Lambda_{j}^{*} f=0$ for all $i \notin \Delta$, in particular, $\Lambda_{j} \Lambda_{j}^{*} f=0$. It follows that $\Lambda_{j}^{*} f=0, \forall f \in H_{j}$. Hence $\Lambda_{j}^{*}=0$, so $\Lambda_{j}=0$ for all $j \notin \Delta$, which implies that the only way to enlarge a normalized tight $g$-frame so that it remains to be a normalized tight $g$-frame is to add zero operators. 


\section{Dilations of $g$-FRAMES}

In this section, the dilations of $g$-frames are studied.

Before we introduce the main results of this section we need the following lemma.

Lemma 4.1. [2] Let $\left\{\Lambda_{i} \in B\left(H, H_{i}\right): i \in \mathcal{N}\right\}$ be a normalized tight g-frame for $H$ with respect to $\left\{H_{i}: i \in \mathcal{N}\right\}$. Then there exists a Hilbert space $\mathcal{K} \supseteq H$ and a g-orthonormal basis $\left\{\theta_{i} \in B\left(\mathcal{K}, H_{i}\right): i \in \mathcal{N}\right\}$ for $\mathcal{K}$ with respect to $\left\{\bar{H}_{i}: i \in \mathcal{N}\right\}$ such that $\Lambda_{i} P=\theta_{i} P$, where $P$ is the orthogonal projection from $\mathcal{K}$ onto $H$.

The following result tells us that normalized tight $g$-frame can be dilated to a $g$-orthonormal basis.

Theorem 4.2. A set $\left\{\Lambda_{i}: i \in \mathcal{N}\right\}$ is a normalized tight g-frame for a Hilbert space $H$ with respect to $\left\{H_{i}: i \in \mathcal{N}\right\}$ if and only if there exists a Hilbert space $M$ and a normalized tight $g$-frame $\left\{\Gamma_{j}: j \in \mathcal{N}\right\}$ for $M$ such that $\left\{\Lambda_{j} \oplus \Gamma_{j}: j \in \mathcal{N}\right\}$ is a g-orthnormal basis for $H \oplus M$.

Proof. $\Rightarrow$ : Since $\left\{\Lambda_{i}: i \in \mathcal{N}\right\}$ is a normalized tight $g$-frame for Hilbert space $H$ with respect to $\left\{H_{i}: i \in \mathcal{N}\right\}$, by Lemma 4.1, there is a larger Hilbert space $\mathcal{K} \supseteq H$ and a $g$-orthonormal basis $\left\{\theta_{j}: j \in \mathcal{N}\right\}$ for $\mathcal{K}$ such that $\Lambda_{j} P=\theta_{j} P$ for each $j \in \mathcal{N}$, where $P$ is the orthogonal projection from $\mathcal{K}$ onto $H$. Let $M=(I-P) \mathcal{K}$ and $\Gamma_{j} \in B\left(M, H_{j}\right)$ such that $\Gamma_{j}(I-P)=\theta_{j}(I-P), \forall j \in \mathcal{N}$. Then $\left\{\Gamma_{j}: j \in \mathcal{N}\right\}$ is a normalized tight $g$-frame for $M$ and $\left\{\Lambda_{j} \oplus \Gamma_{j}: j \in \mathcal{N}\right\}=$ $\left\{\theta_{j} P \oplus \theta_{j}(I-P): j \in \mathcal{N}\right\}$. It is easy to see that $\left\{\theta_{j} P \oplus \theta_{j}(I-P): j \in \mathcal{N}\right\}$ is a $g$-orthonormal basis for $H \oplus M$ by a simple computation.

$\Leftarrow$ : This direction is obvious.

Moreover, the following result shows that the above dilation is unique in the sense of unitary equivalence.

Theorem 4.3. The extension of a normalized tight g-frame to a g-orthonormal basis described in the statement of above is unique up to unitary equivalence. That is if $N$ is another Hilbert space and $\left\{L_{j}: j \in \mathcal{N}\right\}$ is a normalized tight g-frame for $N$ such that $\left\{\Lambda_{j} \oplus L_{j}: j \in \mathcal{N}\right\}$ is a g-orthonormal basis for $H \oplus N$, then there is a unitary transformation $U$ mapping $M$ onto $N$ such that $\Gamma_{j} U^{*}=L_{j}$ for all $j \in \mathcal{N}$.

Proof. Since $\left\{\Lambda_{j} \oplus \Gamma_{j}: j \in \mathcal{N}\right\}$ and $\left\{\Lambda_{j} \oplus L_{j}: j \in \mathcal{N}\right\}$ are $g$-orthonormal bases for $H \oplus M$ and $H \oplus N$ respectively, $\left\{\left(\Lambda_{j} \oplus \Gamma_{j}\right)^{*} g_{j}: g_{j} \in H_{j}, j \in \mathcal{N}\right\}$ is dense in $H \oplus M,\left\{\left(\Lambda_{j} \oplus L_{j}\right)^{*} g_{j}: g_{j} \in H_{j}, j \in \mathcal{N}\right\}$ is dense in $H \oplus N$. Define a unitary operator $\widetilde{U}: H \oplus M \rightarrow H \oplus N$ by

$$
\widetilde{U}\left(\Lambda_{j} \oplus \Gamma_{j}\right)^{*} g_{j}=\left(\Lambda_{j} \oplus L_{j}\right)^{*} g_{j},
$$

and the values of all other points are defined by linearity and continuity of $\widetilde{U}$. Since for any $n \in \mathcal{N}$, we have

$$
\begin{aligned}
& \left(\Lambda_{n} \oplus \Gamma_{n}\right)\left(\Lambda_{j}^{*} g_{j} \oplus 0_{M}\right)=\Lambda_{n} \Lambda_{j}^{*} g_{j}+\Gamma_{n} 0_{M}=\Lambda_{n} \Lambda_{j}^{*} g_{j} \\
& =\Lambda_{n} \Lambda_{j}^{*} g_{j}+L_{n} 0_{N}=\left(\Lambda_{n} \oplus L_{n}\right)\left(\Lambda_{j}^{*} g_{j} \oplus 0_{N}\right),
\end{aligned}
$$


and

$$
\begin{aligned}
& \Lambda_{j}^{*} g_{j} \oplus 0_{M}=\sum_{n \in \mathcal{N}}\left(\Lambda_{n} \oplus \Gamma_{n}\right)^{*}\left(\Lambda_{n} \oplus \Gamma_{n}\right)\left(\Lambda_{j}^{*} g_{j} \oplus 0_{M}\right) \\
& \Lambda_{j}^{*} g_{j} \oplus 0_{N}=\sum_{n \in \mathcal{N}}\left(\Lambda_{n} \oplus \Gamma_{n}\right)^{*}\left(\Lambda_{n} \oplus \Gamma_{n}\right)\left(\Lambda_{j}^{*} g_{j} \oplus 0_{N}\right)
\end{aligned}
$$

It follows that

$$
\begin{aligned}
& \widetilde{U}\left(\Lambda_{j}^{*} g_{j} \oplus 0_{M}\right)=\widetilde{U} \sum_{n \in \mathcal{N}}\left(\Lambda_{n} \oplus \Gamma_{n}\right)^{*}\left(\Lambda_{n} \oplus \Gamma_{n}\right)\left(\Lambda_{j}^{*} g_{j} \oplus 0_{M}\right) \\
& =\sum_{n \in \mathcal{N}}\left(\Lambda_{n} \oplus L_{n}\right)^{*}\left(\Lambda_{n} \oplus \Gamma_{n}\right)\left(\Lambda_{j}^{*} g_{j} \oplus 0_{M}\right) \\
& =\sum_{n \in \mathcal{N}}\left(\Lambda_{n} \oplus L_{n}\right)^{*}\left(\Lambda_{n} \oplus L_{n}\right)\left(\Lambda_{j}^{*} g_{j} \oplus 0_{N}\right) \\
& =\Lambda_{j}^{*} g_{j} \oplus 0_{N} .
\end{aligned}
$$

Thus $\widetilde{U}=I \oplus U$, where $I$ is the identity operator in $B(H)$ and $U$ is a unitary operator in $B(M, N)$. So

$$
\begin{aligned}
& \widetilde{U}\left(\Lambda_{j} \oplus \Gamma_{j}\right)^{*} g_{j}=(I \oplus U)\left(\Lambda_{j} \oplus \Gamma_{j}\right)^{*} g_{j}=(I \oplus U)\left(\Lambda_{j}^{*} \oplus \Gamma_{j}^{*}\right) g_{j} \\
& =\left(\Lambda_{j}^{*} \oplus U \Gamma_{j}^{*}\right) g_{j} .
\end{aligned}
$$

But $\widetilde{U}\left(\Lambda_{j} \oplus \Gamma_{j}\right)^{*} g_{j}=\left(\Lambda_{j} \oplus L_{j}\right)^{*} g_{j}=\left(\Lambda_{j}^{*} \oplus L_{j}^{*}\right) g_{j}$ by the definition of $\widetilde{U}$. Hence $U \Gamma_{j}^{*}=L_{j}^{*}$, which implies that $L_{j}=\Gamma_{j} U^{*}, \forall j \in \mathcal{N}$.

In order to establish the dilation results on general $g$-frames, we need the following simple fact, which can be proved straightforwardly, so we omit the details.

Lemma 4.4. A g-frame is precisely the image of a normalized tight $g$-frame under the right multiplying by a bounded invertible operator.

By using the dilations about normalized tight $g$-frames and the above lemma, we get the following dilation about $g$-frames.

Theorem 4.5. Suppose $\left\{\Lambda_{j}: j \in \mathcal{N}\right\}$ is a g-frame for $H$. Then there exists a Hilbert space $M$ and a normalized tight $g$-frame $\left\{\Gamma_{j}: j \in \mathcal{N}\right\}$ for $M$ such that $\left\{\Lambda_{j} \oplus \Gamma_{j}: j \in \mathcal{N}\right\}$ is a $g$-Riesz basis for $H \oplus M$.

Proof. By Lemma 4.4, there exists a normalized tight $g$-frame $\left\{L_{j}: j \in \mathcal{N}\right\}$ for $H$ and an invertible operator $T$ in $B(H)$ such that $\Lambda_{j}=L_{j} T, \forall j \in \mathcal{N}$. By Theorem 4.2, there is a Hilbert space $M$ and a normalized tight $g$-frame $\left\{\Gamma_{j}: j \in \mathcal{N}\right\}$ for $M$ such that $\left\{L_{j} \oplus \Gamma_{j}: j \in \mathcal{N}\right\}$ is a $g$-orthonormal basis for $H \oplus M$. Then $T \oplus I$ is an invertible operator in $B(H \oplus M)$ such that $\left(L_{j} \oplus \Gamma_{j}\right)(T \oplus I)=L_{j} T \oplus \Gamma_{j}=\Lambda_{j} \oplus \Gamma_{j}$, $\forall j \in \mathcal{N}$. So $\left\{\Lambda_{j} \oplus \Gamma_{j}: j \in \mathcal{N}\right\}$ is a $g$-Riesz basis for $H \oplus M$.

The following theorem is a collection of simple but useful facts about $g$-frames which we may need in the sequel. Since most of them can be proved straightforwardly, we only give the proofs of the last two statements. 
Theorem 4.6. (i) If $\left\{\Lambda_{j}: j \in \mathcal{N}\right\}$ is a g-frame and $T$ is a co-isometry (that is $T^{*}$ is an isometry), then $\left\{\Lambda_{j} T^{*}: j \in \mathcal{N}\right\}$ is a g-frame. Moreover, $\left\{\Lambda_{j} T^{*}: j \in \mathcal{N}\right\}$ is a normalized tight $g$-frame if $\left\{\Lambda_{j}: j \in \mathcal{N}\right\}$ is.

(ii) Suppose that $\left\{\Lambda_{j}: j \in \mathcal{N}\right\}$ and $\left\{\Gamma_{j}: j \in \mathcal{N}\right\}$ are normalized tight g-frames and suppose that $T$ is a linearly bounded operator which satisfies $\Gamma_{j}=\Lambda_{j} T^{*}$, $\forall j \in \mathcal{N}$. Then $T$ is a co-isometry. If $T$ is invertible, then it is unitary.

(iii) If $\left\{\Lambda_{j}: j \in \mathcal{N}\right\}$ and $\left\{\Gamma_{j}: j \in \mathcal{N}\right\}$ are normalized tight g-frames for Hilbert spaces $H$ and $M$ respectively and $\left\{\Lambda_{j} \oplus \Gamma_{j}: j \in \mathcal{N}\right\}$ is a normalized tight $g$ frame for $H \oplus M$. If $\left\{L_{j}: j \in \mathcal{N}\right\}$ is a normalized tight g-frame for $M$ which is unitarily equivalent to $\left\{\Gamma_{j}: j \in \mathcal{N}\right\}$, then $\left\{\Lambda_{j} \oplus L_{j}: j \in \mathcal{N}\right.$ is also a normalized tight $g$-frame for $H \oplus M$.

(iv) If $\left\{\Lambda_{j}: j \in \mathcal{N}\right\}$ is a g-frame which is also a g-basis, then it is a g-Riesz basis.

(v) If $\left\{\Lambda_{j}: j \in \mathcal{N}\right\}$ is both a $g$-Riesz basis and a normalized tight g-frame, then it is a g-orthonormal basis.

Proof. (iv) This statement has been proved in [11, Lemma 2.16]. Now we give a different new proof by using the dilation results. By Theorem 4.5, there is a normalized tight $g$-frame $\left\{\Gamma_{j}: j \in \mathcal{N}\right\}$ for a Hilbert space $M$ such that $\left\{\Lambda_{j} \oplus \Gamma_{j}\right.$ : $j \in \mathcal{N}\}$ is a $g$-Riesz basis for $H \oplus M$. Let $P$ be the orthogonal projection from $H \oplus M$ onto $H$ and let $z \in M$. Since $0_{H} \oplus z \in H \oplus M$, there exists a sequence $\left\{g_{j}: j \in \mathcal{N}\right\}$ with $g_{j} \in H_{j}, \forall j \in \mathcal{N}$, such that $0_{H} \oplus z=\sum_{j \in \mathcal{N}}\left(\Lambda_{j} \oplus \Gamma_{j}\right)^{*} g_{j}$. Then

$$
0_{H}=P\left(0_{H} \oplus z\right)=P \sum_{j \in \mathcal{N}}\left(\Lambda_{j} \oplus \Gamma_{j}\right)^{*} g_{j}=\sum_{j \in \mathcal{N}} \Lambda_{j}^{*} g_{j} .
$$

Since $\left\{\Lambda_{j}: j \in \mathcal{N}\right\}$ is a $g$-basis for $H, g_{j}=0$ for any $j \in \mathcal{N}$. So $z=0$. Hence $M=\{0\}$ which implies that $\left\{\Lambda_{j}: j \in \mathcal{N}\right\}$ is a $g$-Riesz basis for $H$.

(v) By Lemma 2.10, there is an invertible operator $A$ in $B(H)$ such that $\left\{\Lambda_{j} A\right.$ : $j \in \mathcal{N}\}$ is a $g$-orthonormal basis for $H$. Since $\left\{\Lambda_{j}: j \in \mathcal{N}\right\}$ is a normalized tight $g$-frame for $H$, by (ii) we know that $A$ is a unitary operator. Thus $\left\{\Lambda_{j}: j \in \mathcal{N}\right\}$ is a $g$-orthonormal basis for $H$.

The following result shows that in general the dilation of a $g$-frame is unique in the sense of similarity.

Theorem 4.7. Let $\left\{\Lambda_{j}: j \in \mathcal{N}\right\}$ be a g-frame for $H$. Suppose that $\left\{\Gamma_{j}: j \in \mathcal{N}\right\}$ is a g-frame for Hilbert space $M$ and $\left\{L_{j}: j \in \mathcal{N}\right\}$ is a g-frame for Hilbert space $N$ such that $\left\{\Lambda_{j} \oplus \Gamma_{j}: j \in \mathcal{N}\right\}$ and $\left\{\Lambda_{j} \oplus L_{j}: j \in \mathcal{N}\right\}$ are similar to a g-orthonormal basis for $H \oplus M$ and $H \oplus N$ respectively. Then there exists an invertible operator $A \in B(M, N)$ such that $L_{j}=\Gamma_{j} A, \forall j \in \mathcal{N}$.

Proof. Let $T_{1}, T_{2}, S_{1}, S_{2}$ be invertible operators such that $\left\{\left(\Lambda_{j} \oplus \Gamma_{j}\right)\left(T_{1} \oplus T_{2}\right)\right.$ : $j \in \mathcal{N}\}$ and $\left\{\left(\Lambda_{j} \oplus L_{j}\right)\left(S_{1} \oplus S_{2}\right): j \in \mathcal{N}\right\}$ are $g$-orthonormal bases for $H \oplus M$ and $H \oplus N$ respectively. For any $j \in \mathcal{N}$ let $\Lambda_{j} T_{1}=F_{j}, \Gamma_{j} T_{2}=G_{j}, \Lambda_{j} S_{1}=E_{j}$, $L_{j} S_{2}=K_{j}$. Then $\left\{F_{j}: j \in \mathcal{N}\right\},\left\{G_{j}: j \in \mathcal{N}\right\},\left\{E_{j}: j \in \mathcal{N}\right\},\left\{K_{j}: j \in \mathcal{N}\right\}$ are normalized tight $g$-frames. Since $F_{j}=\Lambda_{j} T_{1}=\left(E_{j} S_{1}^{-1}\right) T_{1}=E_{j}\left(S_{1}^{-1} T_{1}\right)$, $\left\{F_{j}: j \in \mathcal{N}\right\}$ is similar to $\left\{E_{j}: j \in \mathcal{N}\right\}$. By Theorem 4.6 (ii), we know that $U=S_{1}^{-1} T_{1}$ is unitary. Thus $F_{j} \oplus K_{j}=\left(E_{j} \oplus K_{j}\right)(U \oplus I)$. Since $U \oplus I$ is a unitary 
operator on $H \oplus N,\left\{F_{j} \oplus K_{j}: j \in \mathcal{N}\right\}$ is a $g$-orthonormal basis for $H \oplus N$. By Theorem 4.3, there exists a unitary operator $V \in B(M, N)$ such that $K_{j}=G_{j} V$ for any $j \in \mathcal{N}$. Thus $L_{j} S_{2}=\Gamma_{j} T_{2} V$ for any $j \in \mathcal{N}$. Let $A=T_{2} V S_{2}^{-1}$, then $L_{j}=\Gamma_{j} A$ for any $j \in \mathcal{N}$.

The last two results in this section are two interesting results which are closely related to the dilations of normalized tight $g$-frames.

Theorem 4.8. Let $\left\{\Lambda_{j}: j \in \mathcal{N}\right\}$ and $\left\{\Gamma_{j}: j \in \mathcal{N}\right\}$ be normalized tight g-frames for Hilbert spaces $H$ and $K$ respectively. If $\left\{\Lambda_{j}: j \in \mathcal{N}\right\}$ and $\left\{\Gamma_{j}: j \in \mathcal{N}\right\}$ are unitarily equivalent, then $\left\{\Lambda_{j} \oplus \Gamma_{j}: j \in \mathcal{N}\right\}$ is not g-complete in $H \oplus K$ with respect to $\left\{H_{j}: j \in \mathcal{N}\right\}$.

Proof. Let $U \in B(H, K)$ be a unitary operator such that $\Lambda_{j} U=\Gamma_{j}$ for any $j \in \mathcal{N}$. Then $U \oplus I$ is a unitary operator from $H \oplus K$ onto $K \oplus K$. Since $\left(\Lambda_{j} \oplus \Gamma_{j}\right)(U \oplus I)=\Lambda_{j} U \oplus \Gamma_{j} I=\Gamma_{j} \oplus \Gamma_{j}$ for any $j \in \mathcal{N}$ and $\left\{\Gamma_{j} \oplus \Gamma_{j}: j \in \mathcal{N}\right\}$ is not $g$-complete in $K \oplus K$ since $x \oplus(-x) \perp \overline{\operatorname{span}}\left\{\left(\Gamma_{j} \oplus \Gamma_{j}\right)^{*} g_{j}: g_{j} \in H_{j}, j \in \mathcal{N}\right\}$ for any $x \in K$ and $x \neq 0$. It follows that $\left\{\Lambda_{j} \oplus \Gamma_{j}: j \in \mathcal{N}\right\}$ is not $g$-complete in $H \oplus K$.

Theorem 4.9. Suppose that $\left\{\Lambda_{j}: j \in \mathcal{N}\right\}$ and $\left\{\Gamma_{j}: j \in \mathcal{N}\right\}$ are normalized tight g-frames for Hilbert spaces $H$ and $K$ respectively. If $\left\{\Lambda_{j} \oplus \Gamma_{j}: j \in \mathcal{N}\right\}$ is a normalized tight $g$-frame sequence, i.e., it is a normalized tight $g$-frame for $\overline{\operatorname{span}}\left\{\left(\Lambda_{j} \oplus \Gamma_{j}\right)^{*} g_{j}: g_{j} \in H_{j}, j \in \mathcal{N}\right\}$, then it is in fact a normalized tight $g$-frame for $H \oplus K$.

Proof. Since $\left\{\Lambda_{j} \oplus \Gamma_{j}: j \in \mathcal{N}\right\}$ is a normalized tight $g$-frame for $\overline{\operatorname{span}}\left\{\left(\Lambda_{j} \oplus\right.\right.$ $\left.\left.\Gamma_{j}\right)^{*} g_{j}: g_{j} \in H_{j}, j \in \mathcal{N}\right\}$, for any fixed $j \in \mathcal{N}$ and $g_{j} \in H_{j}$, we have

$$
\begin{aligned}
& \left(\Lambda_{j} \oplus \Gamma_{j}\right)^{*} g_{j}=\sum_{n \in \mathcal{N}}\left(\Lambda_{n} \oplus \Gamma_{n}\right)^{*}\left(\Lambda_{n} \oplus \Gamma_{n}\right)\left(\Lambda_{j} \oplus \Gamma_{j}\right)^{*} g_{j} \\
& =\sum_{n \in \mathcal{N}}\left(\Lambda_{n} \oplus \Gamma_{n}\right)^{*}\left(\Lambda_{n} \Lambda_{j}^{*} \oplus \Gamma_{n} \Gamma_{j}^{*}\right) g_{j} \\
& =\sum_{n \in \mathcal{N}}\left(\Lambda_{n}^{*} \oplus \Gamma_{n}^{*}\right)\left(\Lambda_{n} \Lambda_{j}^{*} g_{j}+\Gamma_{n} \Gamma_{j}^{*} g_{j}\right) \\
& =\sum_{n \in \mathcal{N}} \Lambda_{n}^{*}\left(\Lambda_{n} \Lambda_{j}^{*} g_{j}+\Gamma_{n} \Gamma_{j}^{*} g_{j}\right) \oplus \sum_{n \in \mathcal{N}} \Gamma_{n}^{*}\left(\Lambda_{n} \Lambda_{j}^{*} g_{j}+\Gamma_{n} \Gamma_{j}^{*} g_{j}\right) .
\end{aligned}
$$

Since $\left\{\Lambda_{j}: j \in \mathcal{N}\right\}$ and $\left\{\Gamma_{j}: j \in \mathcal{N}\right\}$ are normalized tight $g$-frames for $H$ and $K$ respectively, for $\forall j \in \mathcal{N}$ and $g_{j} \in H_{j}$, we have

$$
\Lambda_{j}^{*} g_{j}=\sum_{n \in \mathcal{N}} \Lambda_{n}^{*} \Lambda_{n} \Lambda_{j}^{*} g_{j} \text { and } \Gamma_{j}^{*} g_{j}=\sum_{n \in \mathcal{N}} \Gamma_{n}^{*} \Gamma_{n} \Gamma_{j}^{*} g_{j} .
$$

So

$$
\Lambda_{j}^{*} g_{j} \oplus \Gamma_{j}^{*} g_{j}=\sum_{n \in \mathcal{N}} \Lambda_{n}^{*} \Lambda_{n} \Lambda_{j}^{*} g_{j} \oplus \sum_{n \in \mathcal{N}} \Gamma_{n}^{*} \Gamma_{n} \Gamma_{j}^{*} g_{j} .
$$

It follows that

$$
\sum_{n \in \mathcal{N}} \Lambda_{n}^{*} \Gamma_{n} \Gamma_{j}^{*} g_{j}=0 \text { and } \sum_{n \in \mathcal{N}} \Gamma_{n}^{*} \Lambda_{n} \Lambda_{j}^{*} g_{j}=0
$$


So, for $\forall j \in \mathcal{N}$ and $g_{j} \in H_{j}$, we have

$$
\begin{aligned}
& \Lambda_{j}^{*} g_{j} \oplus 0_{K}=\sum_{n \in \mathcal{N}} \Lambda_{n}^{*} \Lambda_{n} \Lambda_{j}^{*} g_{j} \oplus \sum_{n \in \mathcal{N}} \Gamma_{n}^{*} \Lambda_{n} \Lambda_{j}^{*} g_{j} \\
& =\sum_{n \in \mathcal{N}}\left(\Lambda_{n} \oplus \Gamma_{n}\right)^{*}\left(\Lambda_{n} \oplus \Gamma_{n}\right)\left(\Lambda_{j}^{*} g_{j} \oplus 0_{K}\right) .
\end{aligned}
$$

and

$$
\begin{aligned}
& 0_{H} \oplus \Gamma_{j}^{*} g_{j}=\sum_{n \in \mathcal{N}} \Lambda_{n}^{*} \Gamma_{n} \Gamma_{j}^{*} g_{j} \oplus \sum_{n \in \mathcal{N}} \Gamma_{n}^{*} \Gamma_{n} \Gamma_{j}^{*} g_{j} \\
& =\sum_{n \in \mathcal{N}}\left(\Lambda_{n} \oplus \Gamma_{n}\right)^{*}\left(\Lambda_{n} \oplus \Gamma_{n}\right)\left(0_{H} \oplus \Lambda_{j}^{*} g_{j}\right) .
\end{aligned}
$$

It follows that

$$
\Lambda_{j}^{*} g_{j} \oplus 0_{K}, 0_{H} \oplus \Gamma_{j}^{*} g_{j} \in \overline{\operatorname{span}}\left\{\left(\Lambda_{n} \oplus \Gamma_{n}\right)^{*} g_{n}: n \in \mathcal{N}\right\} .
$$

Now suppose that $x \in H, y \in K$ and $x \oplus y \perp \overline{\operatorname{span}}\left\{\left(\Lambda_{n} \oplus \Gamma_{n}\right)^{*} g_{n}: g_{n} \in H_{n}, n \in\right.$ $\mathcal{N}\}$. Then $x \oplus y \perp \Lambda_{j}^{*} g_{j} \oplus 0_{K}$ and $x \oplus y \perp 0_{H} \oplus \Gamma_{j}^{*} g_{j}, \forall g_{j} \in H_{j}$ and $\forall j \in \mathcal{N}$. So $\left\langle x, \Lambda_{j}^{*} g_{j}\right\rangle=0$ and $\left\langle y, \Gamma_{j}^{*} g_{j}\right\rangle=0, \forall g_{j} \in H_{j}$ and $\forall j \in \mathcal{N}$. But $\overline{\operatorname{span}}\left\{\Lambda_{j}^{*} g_{j}: g_{j} \in\right.$ $\left.H_{j}, j \in \mathcal{N}\right\}=H$ and $\overline{\operatorname{span}}\left\{\Gamma_{j}^{*} g_{j}: g_{j} \in H_{j}, j \in \mathcal{N}\right\}=K$ since $\left\{\Lambda_{j}: j \in \mathcal{N}\right\}$ and $\left\{\Gamma_{j}: j \in \mathcal{N}\right\}$ are $g$-frames for $H$ and $K$ respectively. Hence $x=0_{H}$ and $y=0_{K}$. So $x \oplus y=0_{H} \oplus 0_{K}$. It implies that $\overline{\operatorname{span}}\left\{\left(\Lambda_{n} \oplus \Gamma_{n}\right)^{*} g_{n}: g_{n} \in H_{n}, n \in \mathcal{N}\right\}=H \oplus K$. So $\left\{\Lambda_{n} \oplus \Gamma_{n}: n \in \mathcal{N}\right\}$ is a normalized tight $g$-frame for $H \oplus K$.

\section{Some PROPERTies OF CANONICAL DUALS OF $g$-FRAMES}

In this section, we study the canonical duals of $g$-frames in Hilbert spaces. Some interesting properties are established.

The following result is the main result in this section, which gives a general method to compute the canonical duals of $g$-frames.

Theorem 5.1. Let $\left\{\Lambda_{j}: j \in \mathcal{N}\right\}$ be a g-frame for $H$. Then there exists a unique operator $S \in B(H)$ such that $f=\sum_{j \in \mathcal{N}} \Lambda_{j}^{*} \Lambda_{j} S f$ for all $f \in H$. An explicit formula for $S$ is given by $S=A^{*} A$, where $A$ is any invertible operator in $B(H, K)$ for some Hilbert space $K$ with the property that $\left\{\Lambda_{j} A^{*}: j \in \mathcal{N}\right\}$ is a normalized tight $g$-frame for $K$. In particular, $S$ is an invertible positive operator. ( We will denote $\Lambda_{j} S$ as $\widetilde{\Lambda}_{j}$ in the sequel)

Proof. Let $A$ be any invertible operator in $B(H, K)$ for some Hilbert space $K$ with the property that $\left\{\Lambda_{j} A^{*}: j \in \mathcal{N}\right\}$ is a normalized tight $g$-frame for $K$. Let $\Gamma_{j}=\Lambda_{j} A^{*}, \forall j \in \mathcal{N}$, and let $S=A^{*} A \in B(H)$. Then

$$
\begin{aligned}
& \sum_{j \in \mathcal{N}} \Lambda_{j}^{*} \Lambda_{j} A^{*} A f=\sum_{j \in \mathcal{N}} \Lambda_{j}^{*} \Gamma_{j} A f=\sum_{j \in \mathcal{N}}\left(A^{-1} \Gamma_{j}^{*}\right) \Gamma_{j} A f \\
& =A^{-1} \sum_{j \in \mathcal{N}} \Gamma_{j}^{*} \Gamma_{j} A f=A^{-1}(A f)=f .
\end{aligned}
$$


So $S=A^{*} A$ satisfies the requirement. For the uniqueness, suppose that $T \in$ $B(H)$ satisfies that for any $f \in H, f=\sum_{j \in \mathcal{N}} \Lambda_{j}^{*} \Lambda_{j} T f$. Then

$$
\begin{aligned}
& f=\sum_{j \in \mathcal{N}} \Lambda_{j}^{*} \Lambda_{j} T f=\sum_{j \in \mathcal{N}}\left(A^{-1} \Gamma_{j}^{*}\right)\left(\Gamma_{j}\left(A^{*}\right)^{-1}\right) T f \\
& =A^{-1} \sum_{j \in \mathcal{N}} \Gamma_{j}^{*} \Gamma_{j}\left(A^{*}\right)^{-1} T f=A^{-1}\left(\left(A^{*}\right)^{-1} T f\right) .
\end{aligned}
$$

It follows that $A^{-1}\left(A^{*}\right)^{-1} T=I$. So $T=A^{*} A=S$.

All the following results can be viewed as some applications of the above theorem.

Corollary 5.2. Suppose that $\left\{\Lambda_{j}: j \in \mathcal{N}\right\}$ is a g-frame for $K$ and $T: H \rightarrow K$ is an invertible operator. Then $\widetilde{\Lambda_{j} T}=\widetilde{\Lambda}_{j}\left(T^{-1}\right)^{*}$ for any $j \in \mathcal{N}$. If $T$ is a unitary operator then $\widetilde{\Lambda_{j} T}=\widetilde{\Lambda}_{j} T$ for any $j \in \mathcal{N}$.

Proof. Let $S \in B(H)$ such that $\widetilde{\Lambda}_{j}=\Lambda_{j} S, \forall j \in \mathcal{N}$. Then for any $f \in H$, we have

$$
f=\sum_{j \in \mathcal{N}} \Lambda_{j}^{*} \widetilde{\Lambda}_{j} f=\sum_{j \in \mathcal{N}} \Lambda_{j}^{*} \Lambda_{j} S f=\sum_{j \in \mathcal{N}} \Lambda_{j}^{*} \Lambda_{j}\left(T T^{-1}\right) S f .
$$

So

$$
\begin{aligned}
& T^{*} f=T^{*} \sum_{j \in \mathcal{N}} \Lambda_{j}^{*} \Lambda_{j}\left(T T^{-1}\right) S f \\
& =\sum_{j \in \mathcal{N}} T^{*} \Lambda_{j}^{*} \Lambda_{j}\left(T T^{-1}\right) S f=\sum_{j \in \mathcal{N}}\left(\Lambda_{j} T\right)^{*}\left(\Lambda_{j} T\right) T^{-1} S f .
\end{aligned}
$$

Hence $f=\sum_{j \in \mathcal{N}}\left(\Lambda_{j} T\right)^{*}\left(\Lambda_{j} T\right) T^{-1} S\left(T^{*}\right)^{-1} f$. It follows that

$$
\widetilde{\Lambda_{j} T}=\Lambda_{j} T\left(T^{-1} S\left(T^{*}\right)^{-1}\right)=\Lambda_{j} S\left(T^{*}\right)^{-1}=\widetilde{\Lambda}_{j}\left(T^{*}\right)^{-1}, \forall j \in \mathcal{N} .
$$

In the case that $T$ is unitary, we have that $T^{*}=T^{-1}$. So $\left(T^{*}\right)^{-1}=T$ and then $\widetilde{\Lambda_{j} T}=\widetilde{\Lambda}_{j} T$ for any $j \in \mathcal{N}$.

Theorem 5.3. Let $\left\{\Lambda_{j}: j \in \mathcal{N}\right\}$ be a g-frame for $H$ and let $S$ be the unique positive operator in $B(H)$ such that $\Lambda_{j} S=\widetilde{\Lambda}_{j}$ for any $j \in \mathcal{N}$. Let $P$ be an orthogonal projection in $B(H)$. Then $\widetilde{\Lambda_{j} P}=\widetilde{\Lambda}_{j} P$ for any $j \in \mathcal{N}$ if and only if $P S=S P$.

Proof. $\Rightarrow$ : Since $\left\{\Lambda_{j} P: j \in \mathcal{N}\right\}$ is a $g$-frame for $P H$, by Theorem 5.1, there exists a unique positive operator $T \in B(P H)$ such that $\Lambda_{j} P \cdot T=\widetilde{\Lambda_{j} P}$ for every $j \in \mathcal{N}$. Since $\widetilde{\Lambda_{j} P}=\widetilde{\Lambda}_{j} P$, we have

$$
\Lambda_{j} P \cdot T=\widetilde{\Lambda_{j} P}=\widetilde{\Lambda}_{j} P=\Lambda_{j} S P, \forall j \in \mathcal{N} .
$$

So $(P T)^{*} \Lambda_{j}^{*}=(S P)^{*} \Lambda_{j}^{*}, \forall j \in \mathcal{N}$. Since $\left\{\Lambda_{j}: j \in \mathcal{N}\right\}$ is a $g$-frame for $H$, $\overline{\operatorname{span}}\left\{\Lambda_{j}^{*}\left(H_{j}\right): j \in \mathcal{N}\right\}=H$. It implies that $(P T)^{*}=(S P)^{*}$. Hence $P T=S P$. It follows that $P T=P S P$. Thus $P S P=S P$. Taking adjoint on both sides, we 
get $P S P=P S$. Hence $P S=S P$.

$\Leftarrow$ : Since $S P=P S$, for any $f \in P H$ we have

$$
f=\sum_{j \in \mathcal{N}} \Lambda_{j}^{*} \widetilde{\Lambda}_{j} f
$$

So

$$
\begin{aligned}
& f=P f=\sum_{j \in \mathcal{N}} P \Lambda_{j}^{*} \widetilde{\Lambda}_{j} f=\sum_{j \in \mathcal{N}} P \Lambda_{j}^{*} \widetilde{\Lambda}_{j} P f \\
& =\sum_{j \in \mathcal{N}}\left(\Lambda_{j} P\right)^{*}\left(\Lambda_{j} S\right) P f=\sum_{j \in \mathcal{N}}\left(\Lambda_{j} P\right)^{*}\left(\Lambda_{j} P\right) S f
\end{aligned}
$$

So $\widetilde{\Lambda_{j} P}=\Lambda_{j} P S=\Lambda_{j} S P=\widetilde{\Lambda}_{j} P, \forall j \in \mathcal{N}$.

The following corollary is obvious, we leave the details to readers.

Corollary 5.4. Let $\left\{\Lambda_{j}: j \in \mathcal{N}\right\}$ be a g-frame for $H$. Then $\left\{\Lambda_{j}: j \in \mathcal{N}\right\}$ is a tight $g$-frame if and only if $\widetilde{\Lambda_{j} P}=\widetilde{\Lambda}_{j} P, \forall j \in \mathcal{N}$ and for all orthogonal projections $P \in B(H)$.

The last result reveals an interesting property about the duals of a $g$-frame, i.e., different duals of a $g$-frame are never similar.

Theorem 5.5. Suppose that $\left\{\Lambda_{j}: j \in \mathcal{N}\right\}$ is a g-frame and $\left\{\Gamma_{j}: j \in \mathcal{N}\right\}$ is an alternate dual g-frame for $\left\{\Lambda_{j}: j \in \mathcal{N}\right\}$. If $T \in B(H)$ is an invertible operator such that $\left\{\Gamma_{j} T: j \in \mathcal{N}\right\}$ is also a dual g-frame of $\left\{\Lambda_{j}: j \in \mathcal{N}\right\}$, then $T=I$. So different dual $g$-frames of a given $g$-frame never similar.

Proof. Since for every $f \in H$, we have

$$
T f=\sum_{j \in \mathcal{N}} \Lambda_{j}^{*} \Gamma_{j} T f=f .
$$

The first equality is based on the fact that $\left\{\Gamma_{j}: j \in \mathcal{N}\right\}$ is a dual $g$-frame of $\left\{\Lambda_{j}: j \in \mathcal{N}\right\}$. The second equality is based on the fact that $\left\{\Gamma_{j} T: j \in \mathcal{N}\right\}$ is a dual $g$-frame of $\left\{\Lambda_{j}: j \in \mathcal{N}\right\}$. So $T=I$.

Acknowledgement. The author would like to thank the referees for valuable comments and suggestions which improved the quality of the paper.

\section{REFERENCES}

1. W. Arveson, Dilation theory yesterday and today, Oper. Theory Adv. Appl. 207 (2010), 99-123.

2. A. Abdollahi and E. Rahimi, Generalized frames on supper Hilbert spaces, Bull. Malays. Math. Sci. Soc. (2) 35(3) (2012), 807-818.

3. P.G. Casazza, The art of frame theory, Taiwanese J. Math. 4(2) (2000), 129-201.

4. C.K. Chui, An Introduction to Wavelets, Acad. Press, New York, 1992.

5. P.G. Casazza, D. Han, and D.R. Larson, Frames for Banach spaces, Contemp. Math. 247 (1999), 149-182.

6. R.J. Duffin and A.C. Shaffer, A class of nonharmonic Fourier Series, Trans. Amer. Math. Soc. 72 (1952), 341-366. 
7. I. Daubechies, Ten Lectures on Wavelets, CBMS-NSF Regional Conference Series in Applied Mathematics, 61. Society for Industrial and Applied Mathematics (SIAM), Philadelphia, PA, 1992.

8. M.L. Ding and Y.C. Zhu, g-Besselian Frames in Hilbert Spaces, Acta Math. Sin. 26 (2010), $2117-2130$.

9. X. Guo, g-Orthonormal bases in Hilbert spaces (in Chinese), Sci. China Ser. A 43 (2013), $1047-1058$.

10. X. Guo, Some properties of g-frames (in Chinese), Acta Math. Sin. 57 (2014), No. 5, $881-892$.

11. X. Guo, g-Bases in Hilbert spaces, Abstr. Appl. Anal., Volume 2012, Article ID 923729, 14 pages, doi:10.1155/2012/923729.

12. D.W. Hadwin, Dilations and Hahn decompositions for linear maps, Canad. J. Math. 33 (1981), 826-839.

13. D. Han, Dilations and completions for Gabor systems, J. Fourier Anal. Appl. 15 (2009), 201-217.

14. D. Han and D.R. Larson, Frames, bases and group representations, Mem. Amer. Math. Soc. 147 (2000), no. 697, 94 pp.

15. E. Hernaandez and G. Weiss, A First Course on Wavelets, CRC press, Boca Raton, FL, 1996.

16. A. Khosravi and K. Musazadeh, Fusion frames and g-frames, J. Math. Anal. Appl. 342 (2008), 1068-1083.

17. S.S. Karimizad, G-Frames, g-orthonormal bases and g-Riesz bases, J. Linear Topol. Algebr. 02 (2013), No. 01, 25-33.

18. J.Z. Li and Y. C. Zhu, Exact g-frames in Hilbert spaces, J. Math. Anal. Appl. 374 (2011), 201-209.

19. S. Mallat, Multiresolution approximations and wavelet orthonormal basis of $L^{2}(R)$, Trans. Amer. Math. Soc. 315 (1989), 69-87.

20. A. Najati, M.H. Faroughi and A. Rahimi, G-frames and stability of g-frames in Hilbert spaces, Methods Funct. Anal. Topology 14 (2008), No. 3, 271-286.

21. B. Sz-Nagy, Expansion Theorems of Paley-Wiener type, Duke Math. J. 14 (1947), 975-978.

22. W.C. Sun, G-frames and g-Riesz bases, J. Math. Anal. Appl. 322 (2006), 437-452.

23. Y.J. Wang and Y.C. Zhu, G-Frames and g-Frames Sequences in Hilbert Spaces, Acta Math. Sin. 25 (2009), No. 12, 2093-2106.

24. R.M. Young, An introduction to nonharmonic Fourier series, Academic Press, New York, 1980.

25. Y.C. Zhu, Characterizations of g-Frames and g-Riesz Bases in Hilbert Spaces, Acta Math. Sin. 24 (2008), 1727-1736.

26. L.L. Zang, W.C. Sun and D.F. Chen, Excess of a class of g-frames, J. Math. Anal. Appl. 352 (2009), 711-717.

Department of Mathematics, Southwestern University of Finance and Economics, Chengdu 611130, P.R. China.

E-mail address: guoxunxiang@yahoo.com 